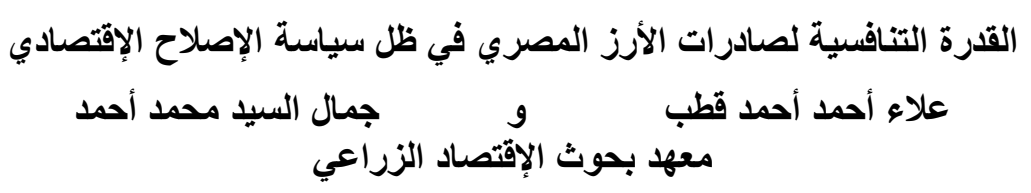

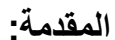

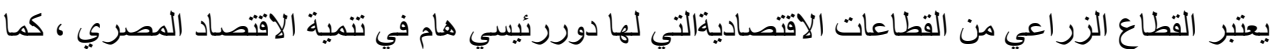

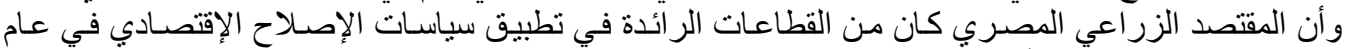

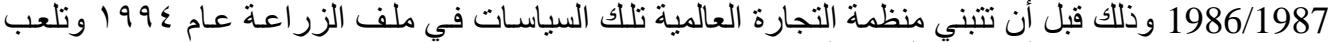

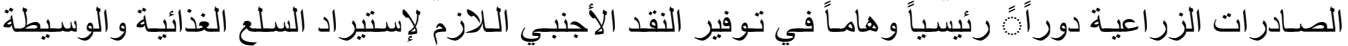
اللازمة لعملية التنمية الإقتصادية.

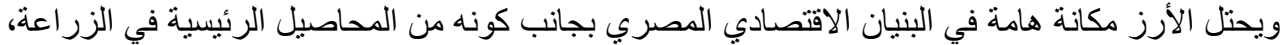

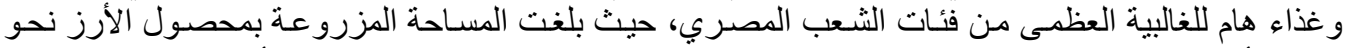

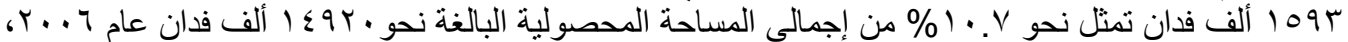

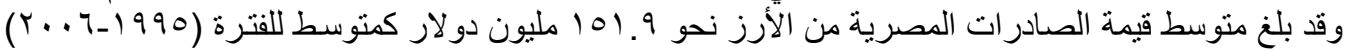

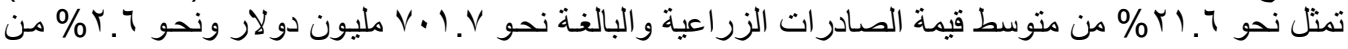

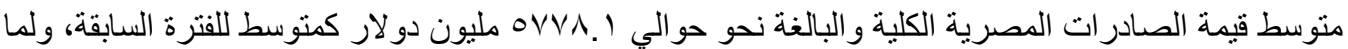

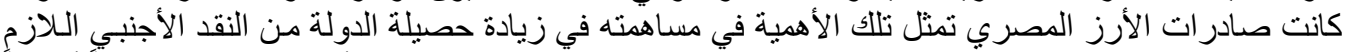

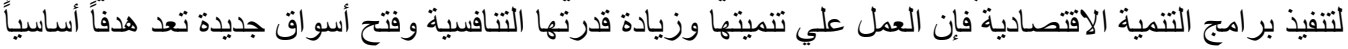

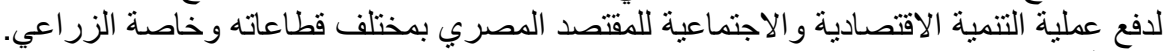
مشكلة البحث: - مئه

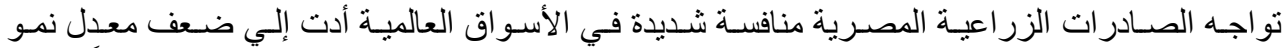

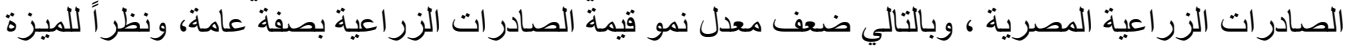

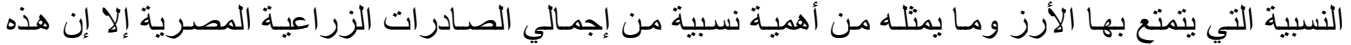

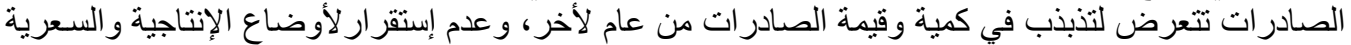

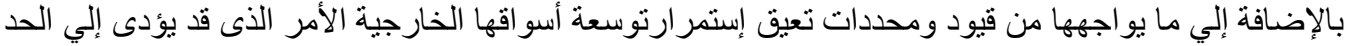

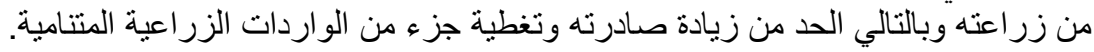

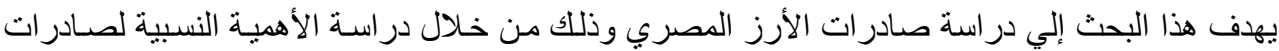

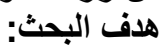

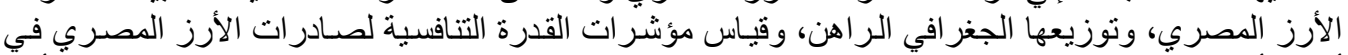

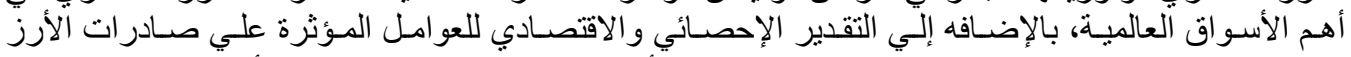

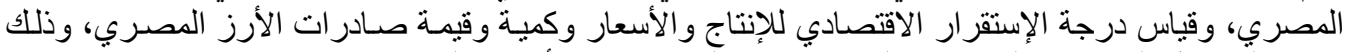

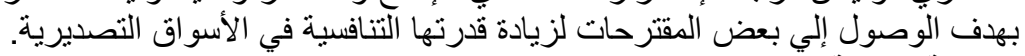
الطريقة البحثية ومصادر البيانات:

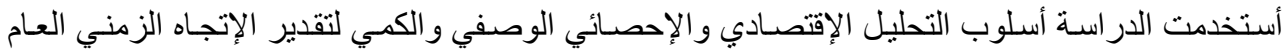

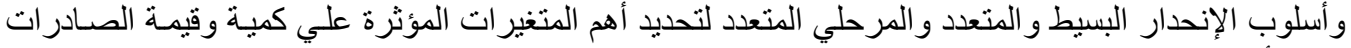

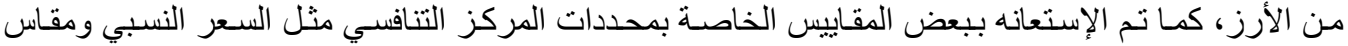

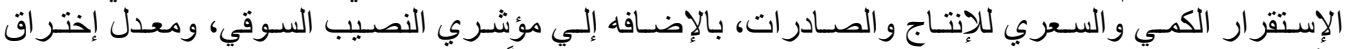

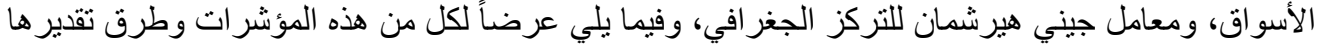

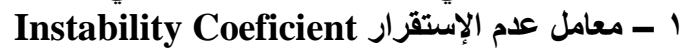

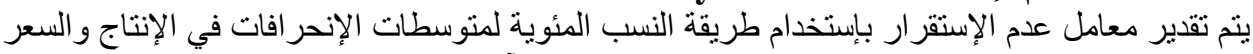
التصديري وكمية وقيمة الصادر ات وذلك من خلال المعادلة الآتية :

$$
\text { معامل عدم الإستقرار = }
$$

حيث ص : تمثل القيم الفعلية للإنتاج أو السعر أو كمية أو قيمة الصادرات من الأرز

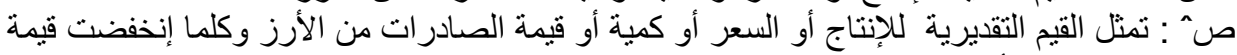

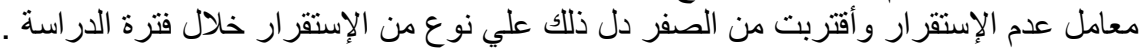

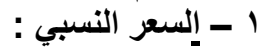

Fayoum J. Agric. Res. \& Dev., Vol.23, No.1, January, 2009 
$\checkmark \varepsilon$

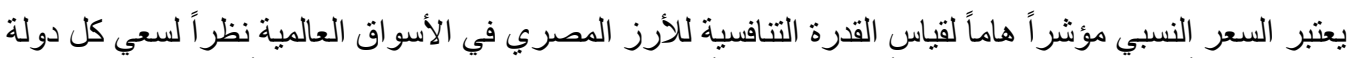

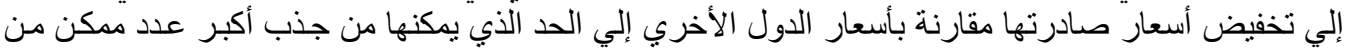

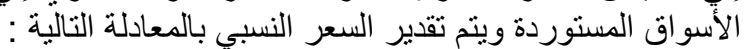

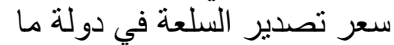
السعر النسبي =

ب - النصيب السوقي Market Share

النصيب السوقي عبارة عن النسبة المئوية لصادرات الدولـة المصدرة للسلعة من إجمالي واردات الدولـة

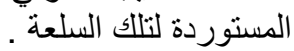

؛ - معدل إختراق السوق العنة

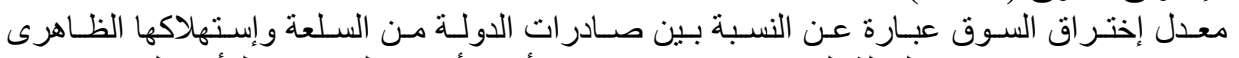

Apparent Consumption

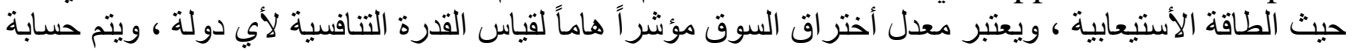
MPRij $=\frac{\text { EXij }}{\text { Qij + Mij - Xij }} \quad * 100 \quad \rightarrow(3)$

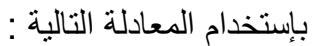

$$
\begin{aligned}
& \text { j معدل إختراق الدولة لأهم الأسواق من السلعة = MPRij }
\end{aligned}
$$

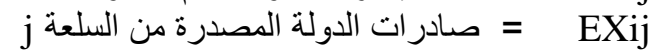

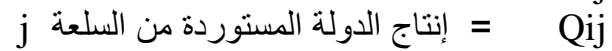

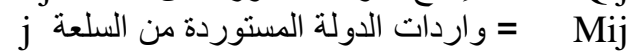

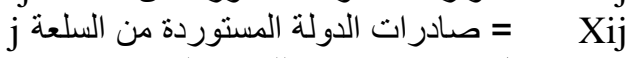

$$
\begin{aligned}
& \text { O معامل جيني هيرشمان للتركز الجغر افي : }
\end{aligned}
$$

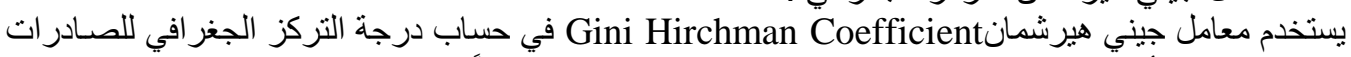
$(\varepsilon) \longleftarrow$

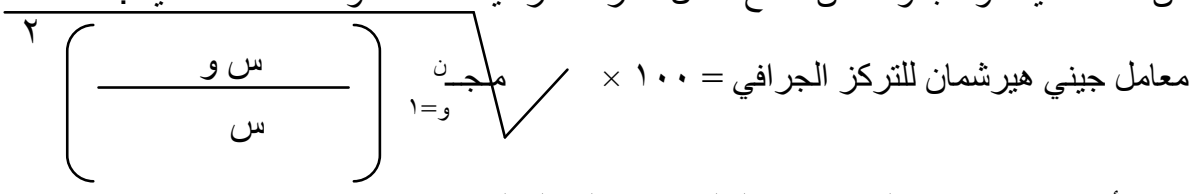

حيث أن س و = كمية الصادر من السلعة (س) إلي الدولة (و)

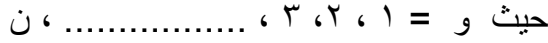

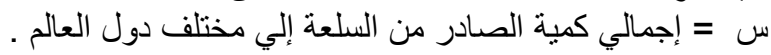

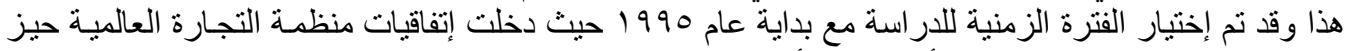

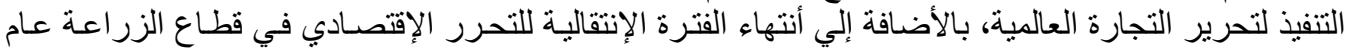

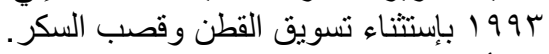

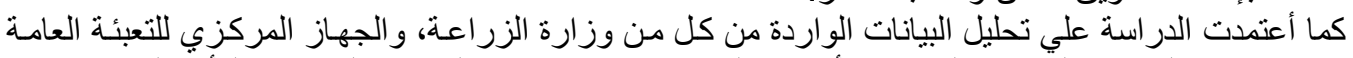

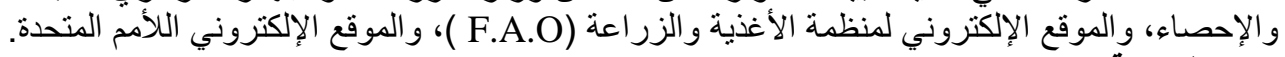

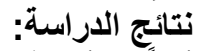

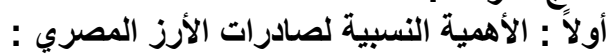
أ - الأهمية النسبية لقيمة صادرات الارزات الألرز المصرية

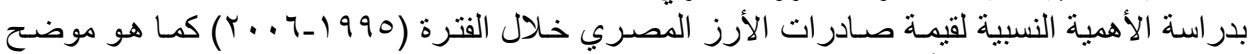

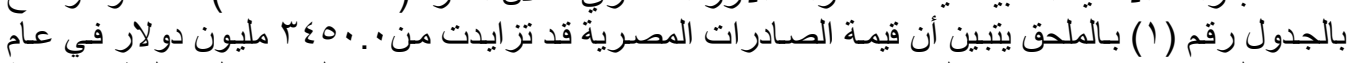

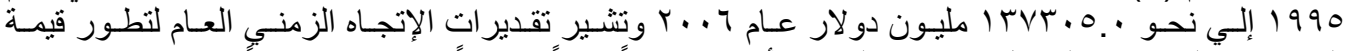

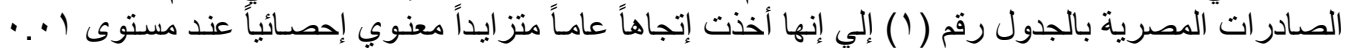

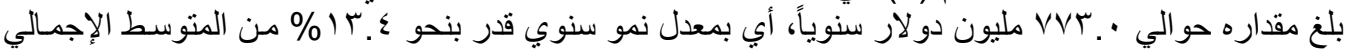

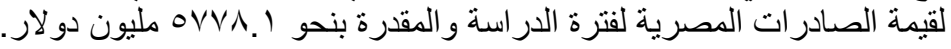

Fayoum J. Agric. Res. \& Dev., Vol.23, No.1, January, 2009 


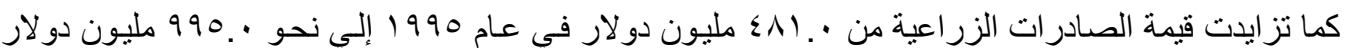

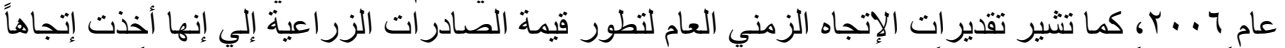

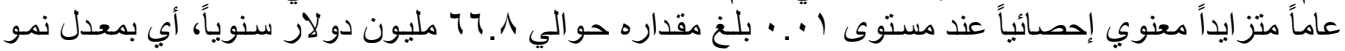

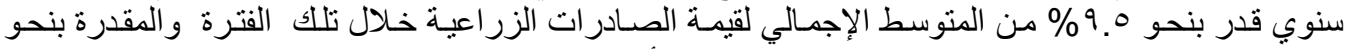

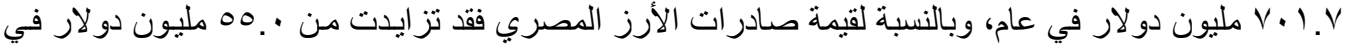

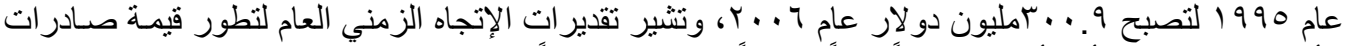

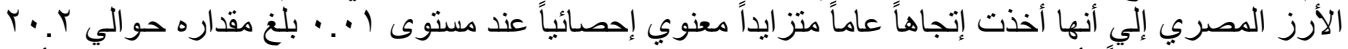

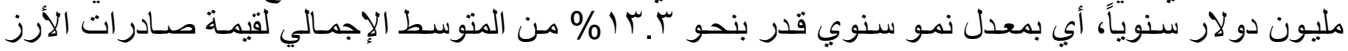

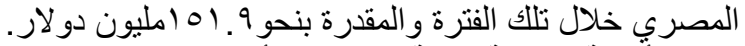

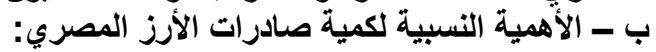

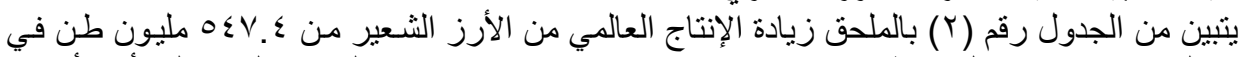

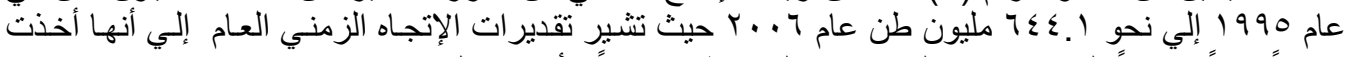

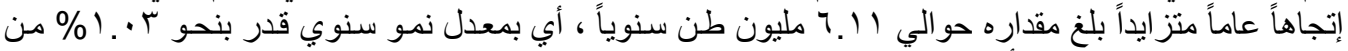

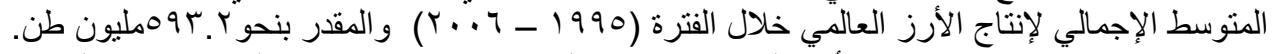

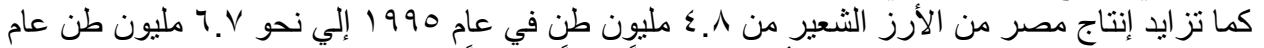

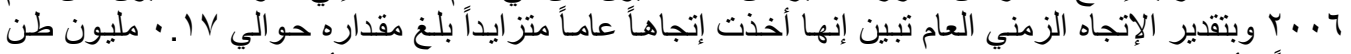

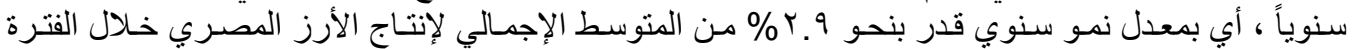

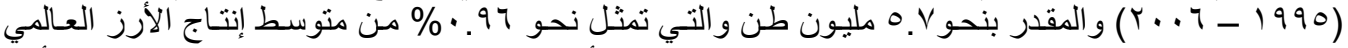

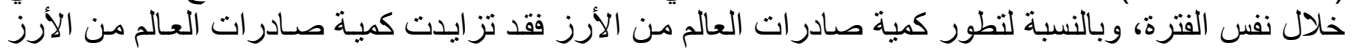

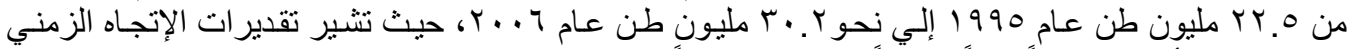

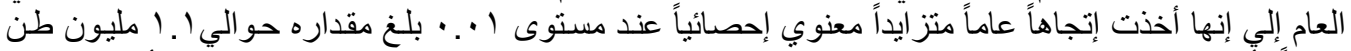

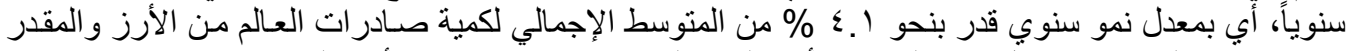

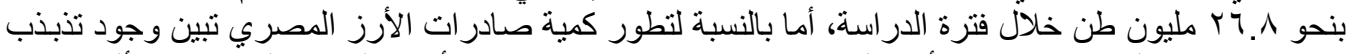

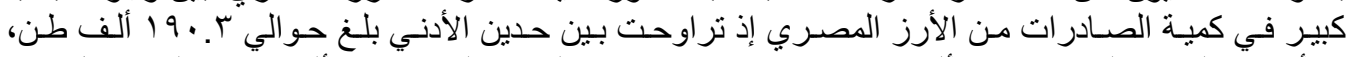

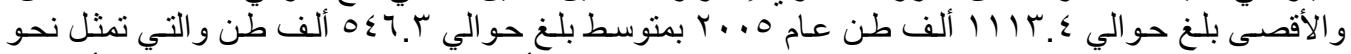

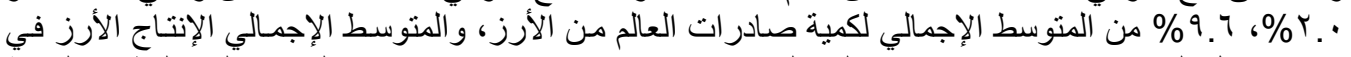

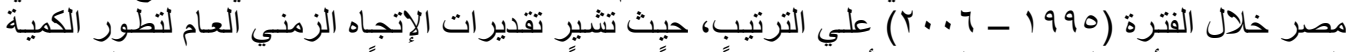

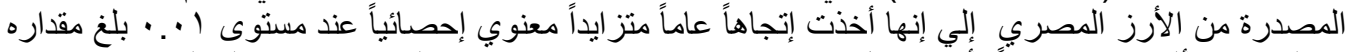

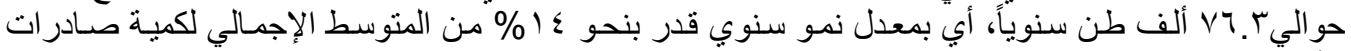

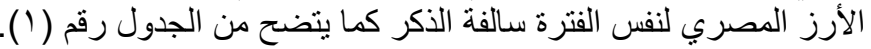

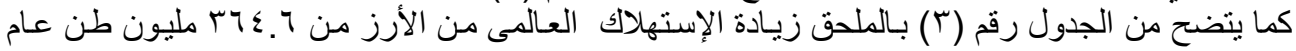

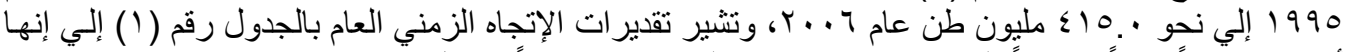

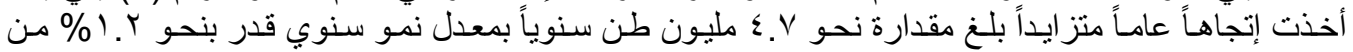

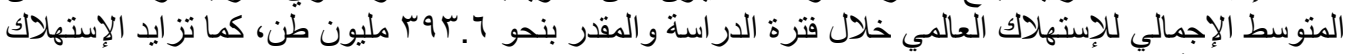

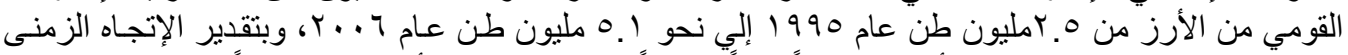

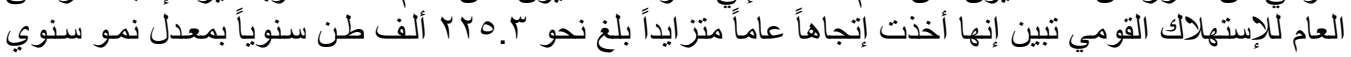

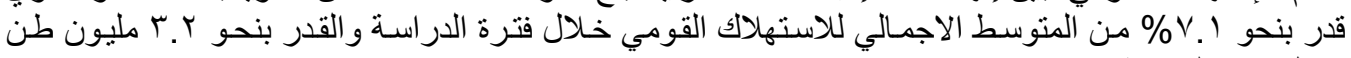

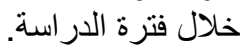

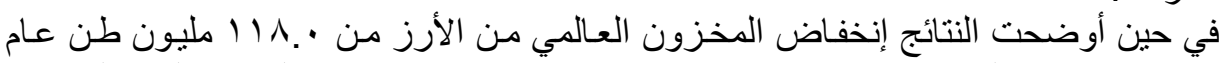

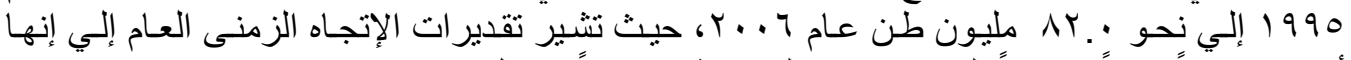

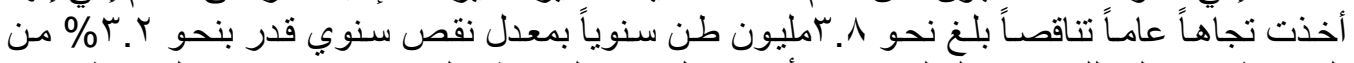

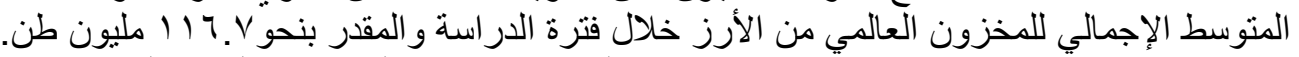

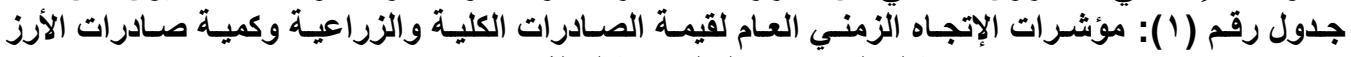

\begin{tabular}{|c|c|c|c|c|c|c|c|}
\hline قلمعامل (ت) & 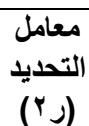 & السنوي النمو & متتوسط & معادلات الإتجاه الزمني العام & والقياس & المتغيرات & لمعادلة \\
\hline$* * \varepsilon .9$ & $\cdot v^{v}$ & $1 \% . \varepsilon$ & OVVA.1 & ص^ه = \&. & 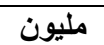 & ق قيمة الصادرات & $T$ \\
\hline
\end{tabular}

Fayoum J. Agric. Res. \& Dev., Vol.23, No.1, January, 2009 


\begin{tabular}{|c|c|c|c|c|c|c|c|}
\hline & & & & & 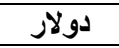 & المصرية الكلية & \\
\hline$* * 0 . r$ & $\cdot V \leqslant$ & 9.0 & $v \cdot 1 . v$ & ص^ ه = ؛. & مليون & المصرية الصادراتة الزراعية & $r$ \\
\hline$* * 0 . T$ & $\cdot V \varepsilon$ & $1 \% . r$ & 101.9 & ص ص ه = = & مليون & قيمة صادرات الأرز & $r$ \\
\hline$* * \leqslant .1$ & .97 & 1.4 & OQY.r & ص هـ = 0. & مليون & كمية الإنتاج العالمي & $\varepsilon$ \\
\hline$* * 0$. & $\cdot .11$ & r.q & $0 . V$ & 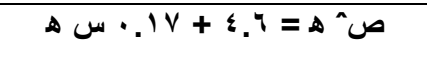 & مليون & كمية الإنتاج المصري) & 0 \\
\hline$* * \mu .0$ & .00 & $\varepsilon .1$ & Y४.A & ص ه = = 19.9 +1.1 س ه & طليون & كمية صادرات الأرز & 7 \\
\hline$* *$ *.^ & $\cdot \Delta r$ & $1 \leqslant$. & $0 \leq 7 . r$ & ص ه = ع . •. + + & ألف طن & كمية صادرات الأرز & $\mathrm{V}$ \\
\hline$* * 1 \cdot . V$ & $\cdot .94$ & $1 . r$ & rq4.q & 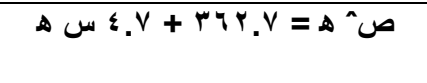 & مليون & كمية الاستثهلاك & $\Lambda$ \\
\hline$* * \leqslant, q$ & $\cdot V$. & V.1 & r191.1 & 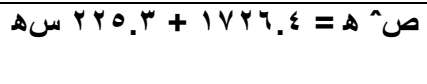 & ألف طن & كمية الاستهلاك المصري & 9 \\
\hline Y.Y. & $\cdot r r$ & T.Y & 117.8 & ص ص^ = = & مليون & كمية المخزون العالمي للارز & 1. \\
\hline$\cdot .74$ & $\because \cdot \varepsilon$ & $\cdot . \varepsilon$ & 10.11 & ص ص ه = V. & مليون & المسالرزة في المنزرعة & 11 \\
\hline 1.8 & $\cdot .1 \mathrm{~V}$ & $\because .9$ & $T \leqslant V \leqslant . r$ & ص^ ه = ".. & ألف فدان & المسأرز في مصر المنزرعة & Ir \\
\hline
\end{tabular}

ص^ هـ : تشير إلي القيمة التقديرية للمتغير ات موضع الدراسة، س هـ: تشير إلي متغير الزمن خلال فترة الدر اسة ن

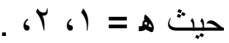

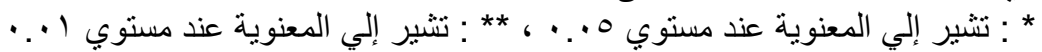

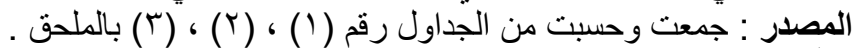

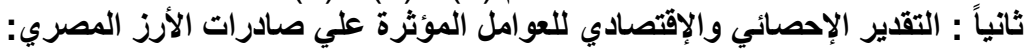

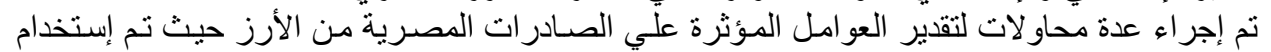

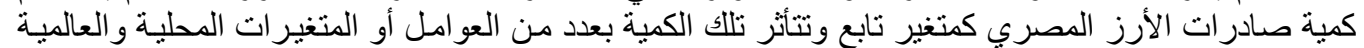

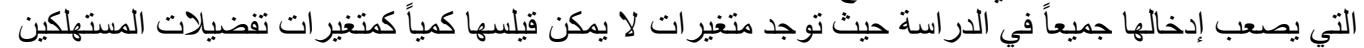

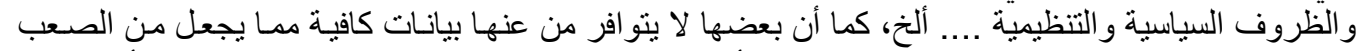

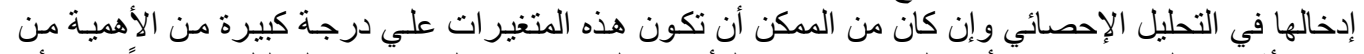

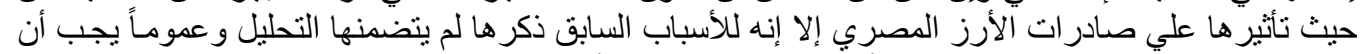

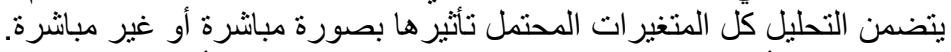

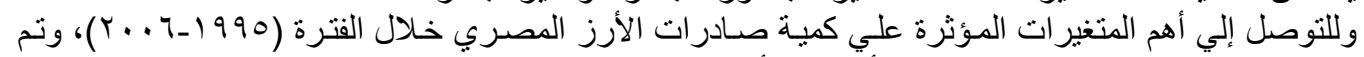

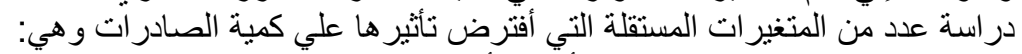

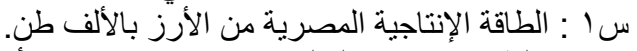

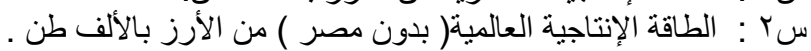

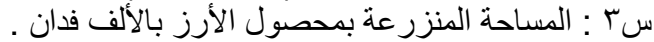

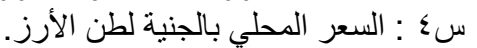

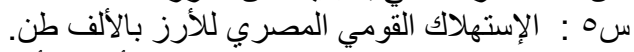

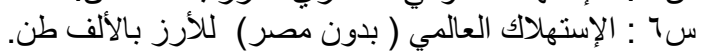

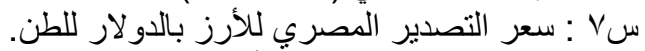

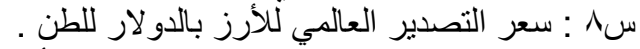

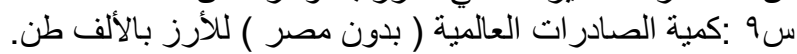

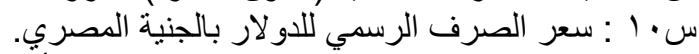

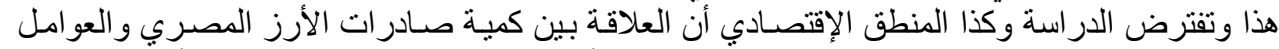

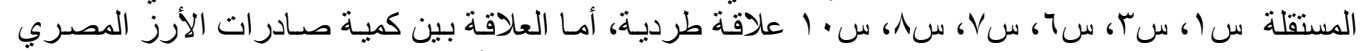

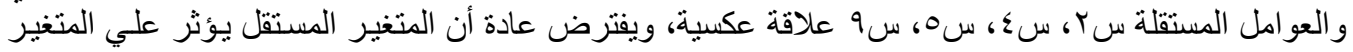

Fayoum J. Agric. Res. \& Dev., Vol.23, No.1, January, 2009 
التابع من خلال نوعين من التأثير أولهما تأثير المتغير المستقل منفرداً علي المتغير التابع وثانيهها تأثيثر المتغير التئير

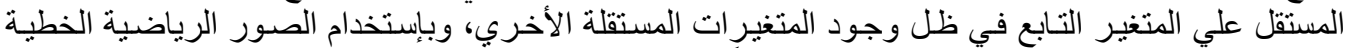

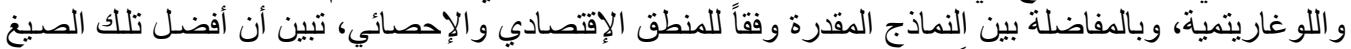

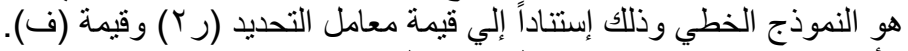

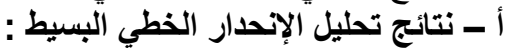

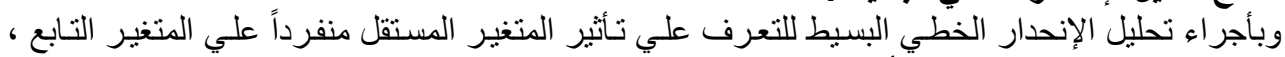

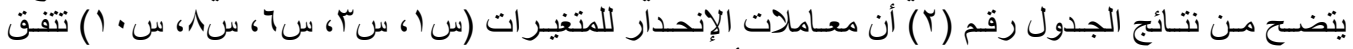

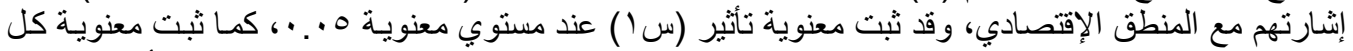

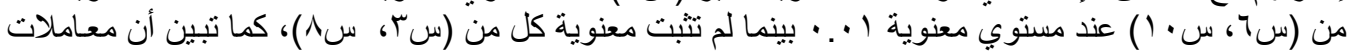

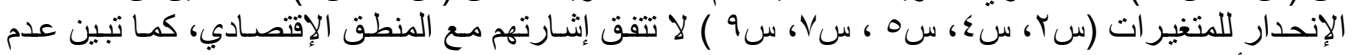

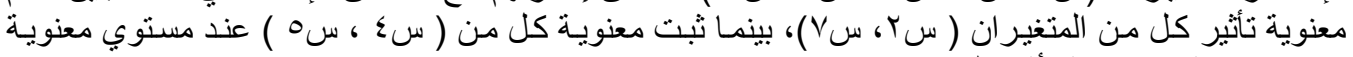

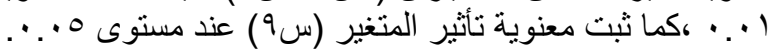

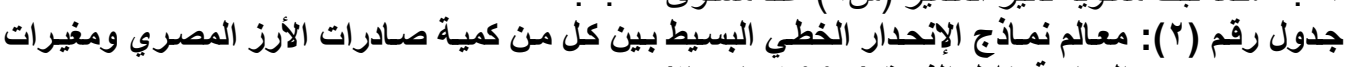

\begin{tabular}{|c|c|c|c|c|c|}
\hline \\
\hline قيمة (ت) & لمعامل الإنحداري & التحديد & معامل الإنحدار & الثابت & المغيــــر \\
\hline${ }^{*}$ Y. $0_{0}$ &. .11 & $\cdot . \mu \Lambda$ & ..$T T$ & $9 \leqslant 9.1-$ & س - س \\
\hline - & $\because \cdots r$ & $\because . \leqslant$ & $\because 10$ & एT\&..- & سץr \\
\hline.$r q$ & .10 & $\because . r$ & 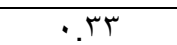 & or.so & سץ \\
\hline$* * 4.1 \leqslant$ & $\cdot r V$ & $.1 \times 9$ & 1.79 & IMAr. & س س س \\
\hline$* * ฯ . \mu 1$ & $\because \bullet$ & $\cdot \wedge$. & - Y^ & $r \leqslant \wedge .7-$ & س0 \\
\hline$* * \Gamma .71$ & $\because \cdots \varepsilon$ & .07 &. $.1 \%$ & $\leqslant \pi r V .7-$ & س7 \\
\hline $1.1 \Lambda_{-}$ & $r . r r$ &. $.1 Y$ & r.rA- & IYYT.T & س V \\
\hline 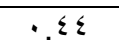 & 1.11 & $\because . r$ & $\cdot . \wedge \varepsilon$ & TVO.V & سـ \\
\hline *Y.qY & $\theta .1 \leqslant$ & $\cdot . \leqslant 7$ & $\because \cdot \varepsilon r$ & $001.7-$ & س99 ( ) \\
\hline$* * \varepsilon, Y Y$ & $\because \leqslant 0$ & .79 & YIY.O & $r \wedge v_{.} \cdot-$ & س.1. \\
\hline
\end{tabular}

ب ـ نتائج تحليل الإنحدار الخطي المتعدد :

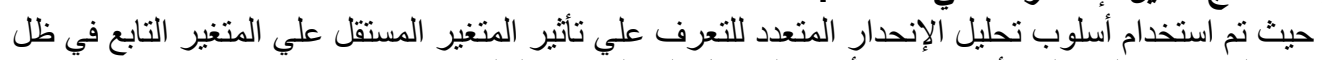

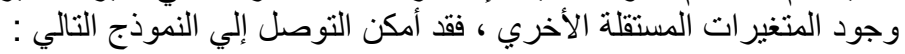

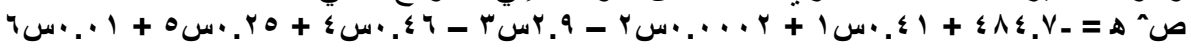

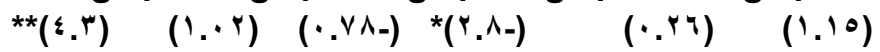

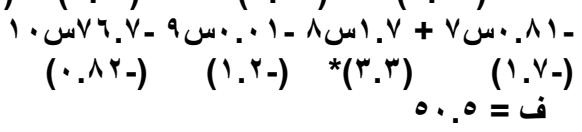

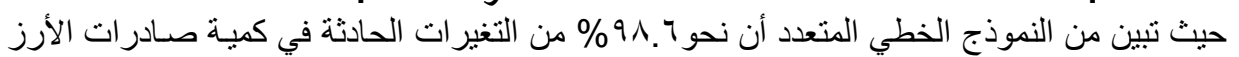

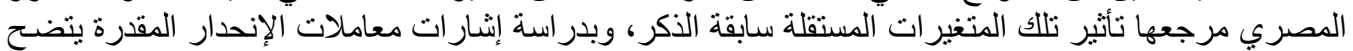

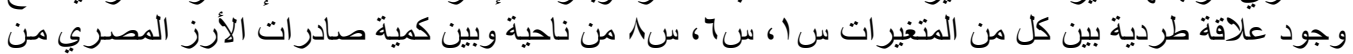

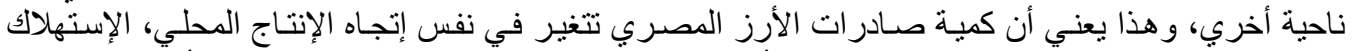

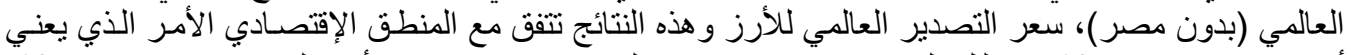

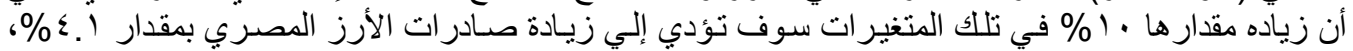

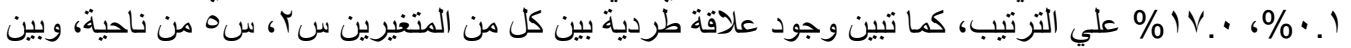

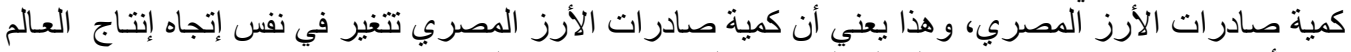

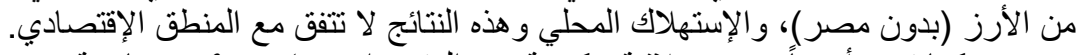

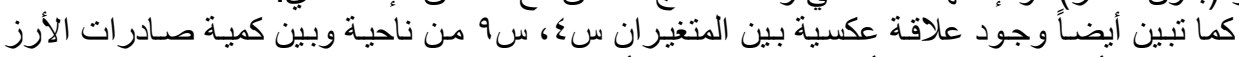

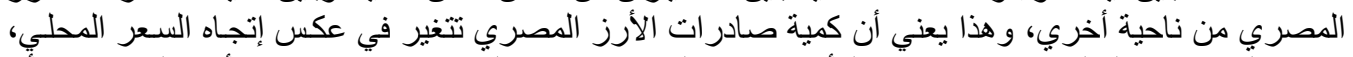

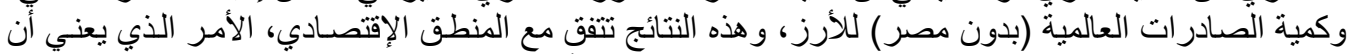

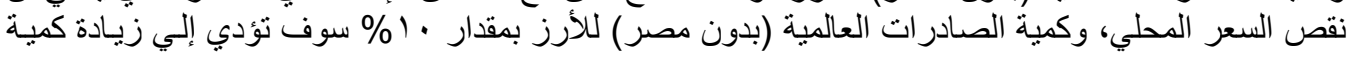

Fayoum J. Agric. Res. \& Dev., Vol.23, No.1, January, 2009 


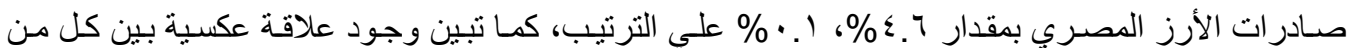

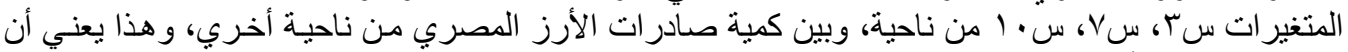

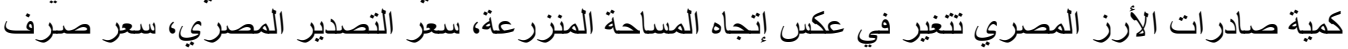

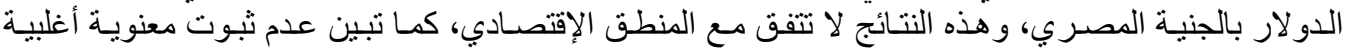

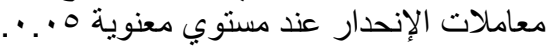
كما تم إستخدام أسلوب تحليل الإنحدار الأنتقائي المرحلي (Step -wise) لتحديد أهم العو امل تأثير أ علي

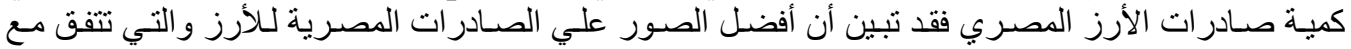
المنطق الإقتصادي والإحصائي تتخذ الصيغة الخطية التالية:

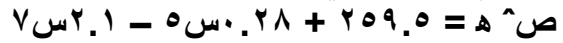
$\left.*\left(r^{\prime} \Lambda_{-}\right) \quad * *\left(\Lambda^{\prime} \cdot\right)^{\prime}\right)$ $\therefore$ ف.$\wedge$ =

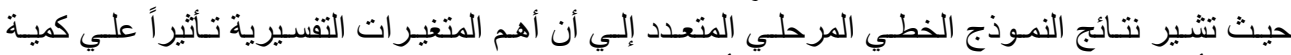

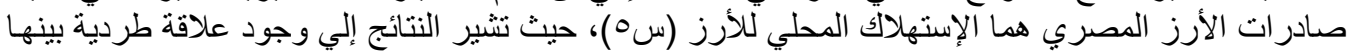

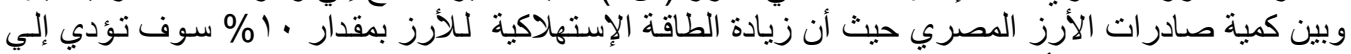

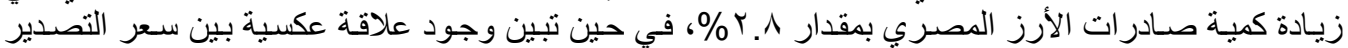

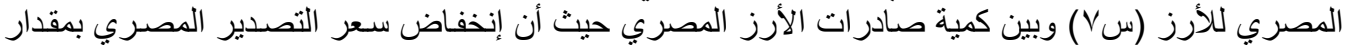

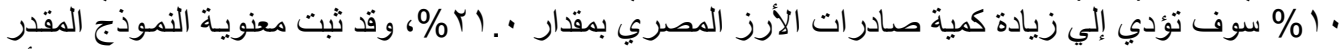

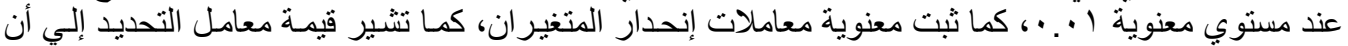

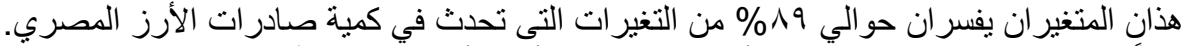

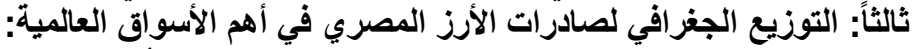

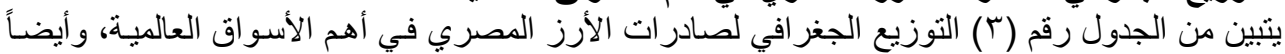

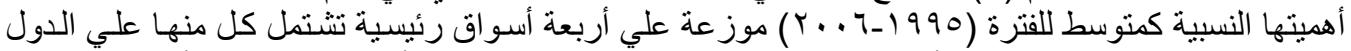

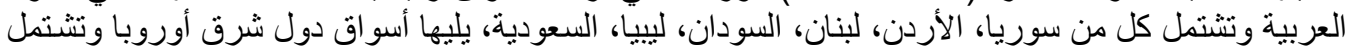

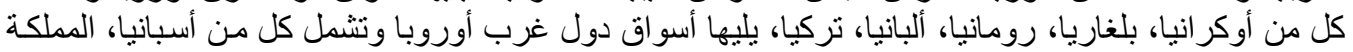

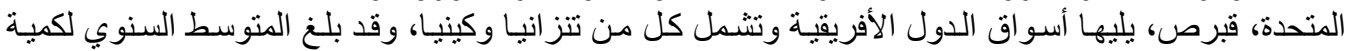

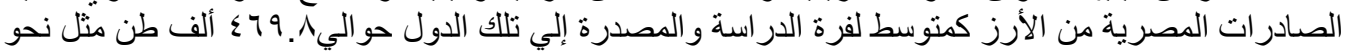

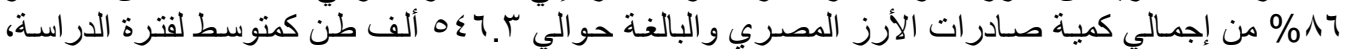

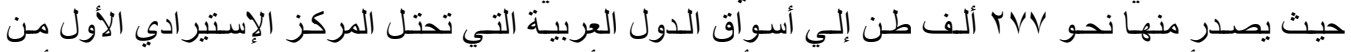

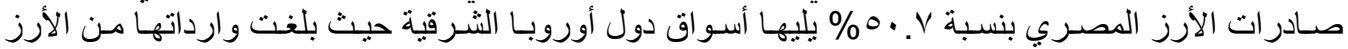

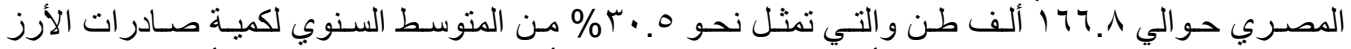

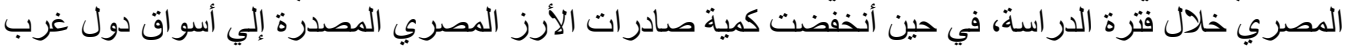

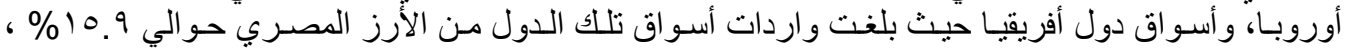

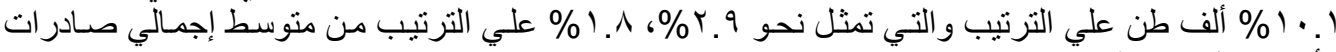

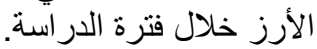

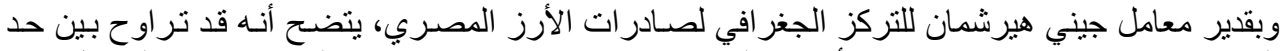

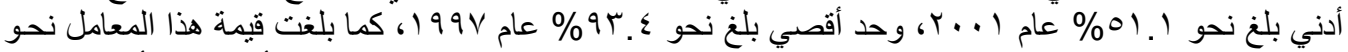

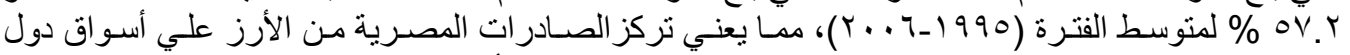

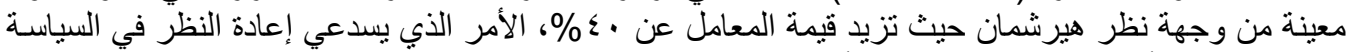

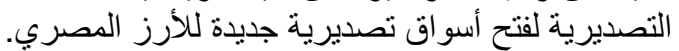

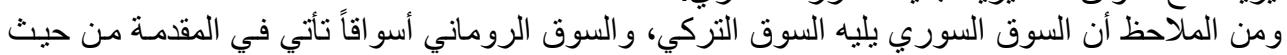

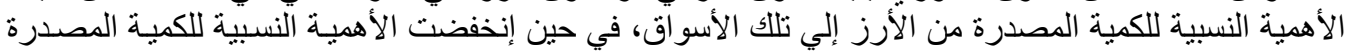

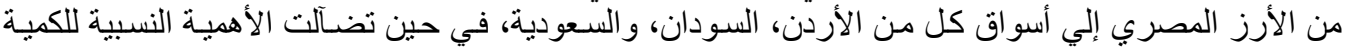

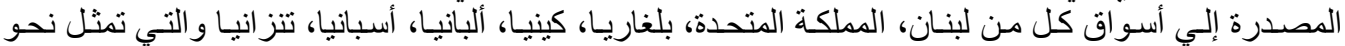

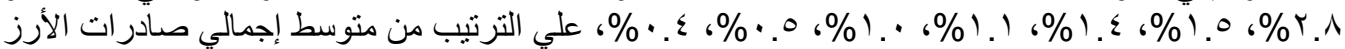

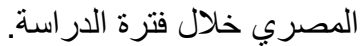

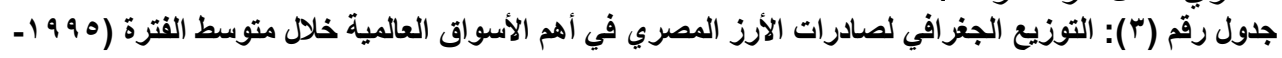

الأسواق وأهم الدول المستوردة

Fayoum J. Agric. Res. \& Dev., Vol.23, No.1, January, 2009 
$\vee 9$

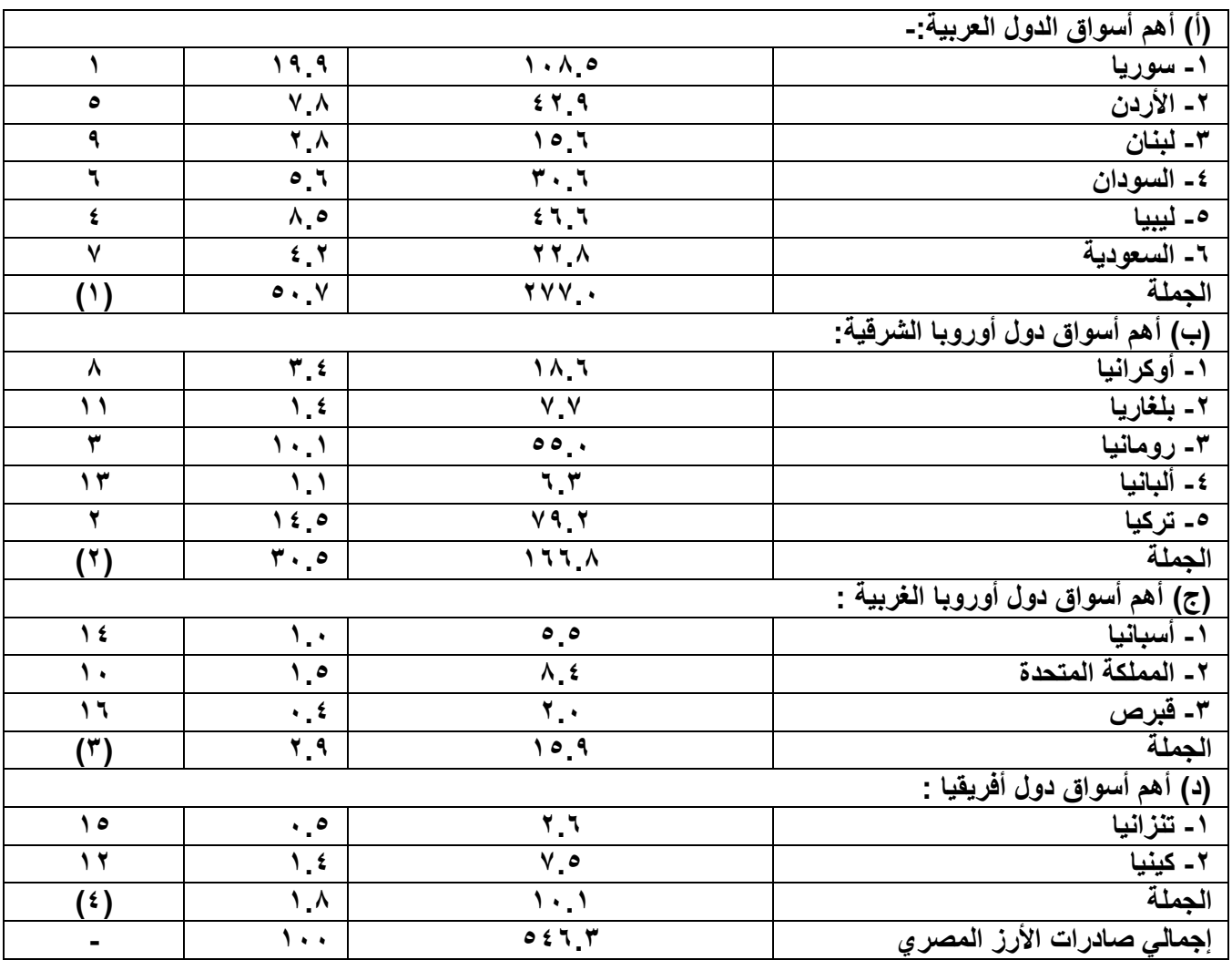

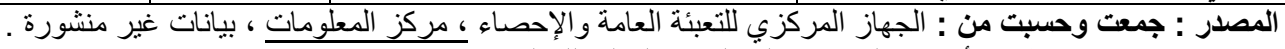

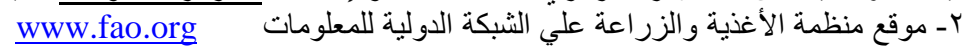

رابعاً : القدرة التتافسية ومحددات المركز التنافسي لصادرات الأرز المصري:

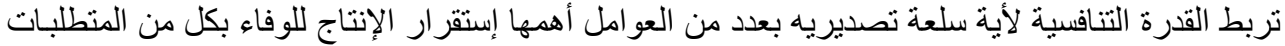

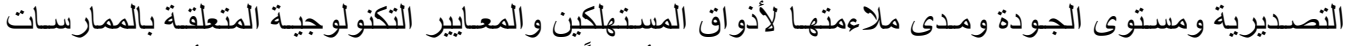

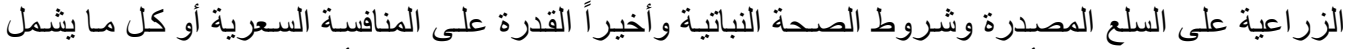

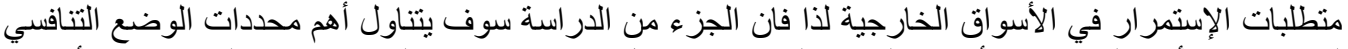

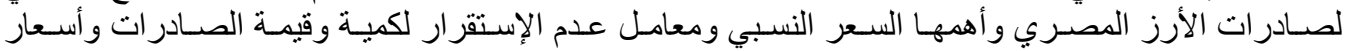

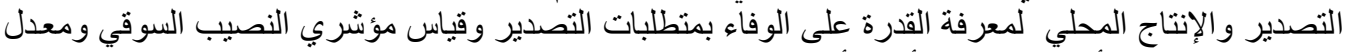

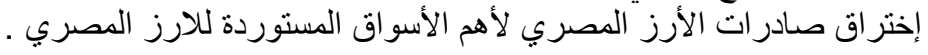

(1) (الإستقرار الكمي والسعرى لصادرات الأرز المصري:

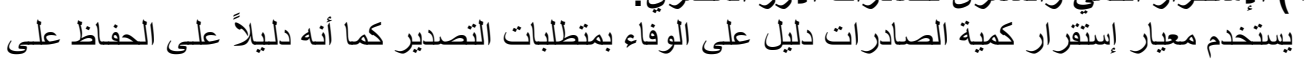

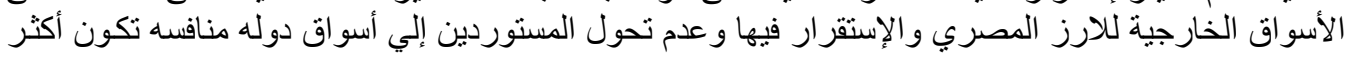

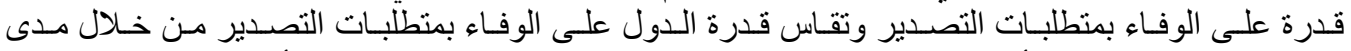

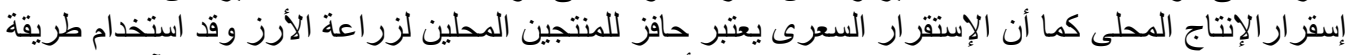

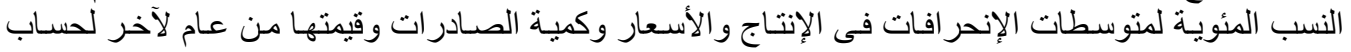

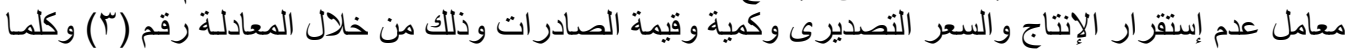

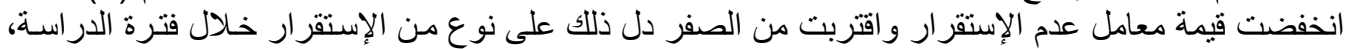

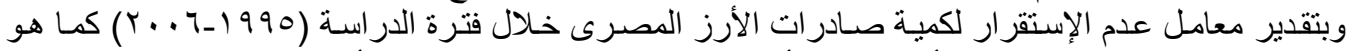

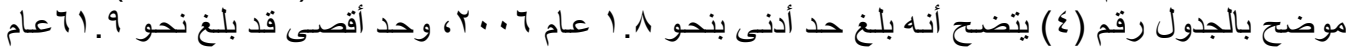

Fayoum J. Agric. Res. \& Dev., Vol.23, No.1, January, 2009 
$\Lambda$.

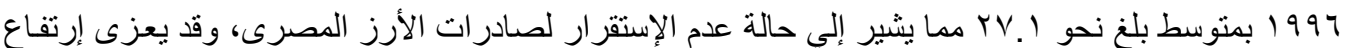

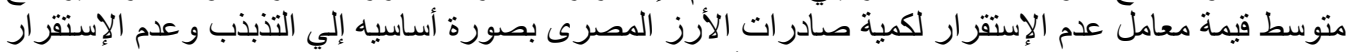

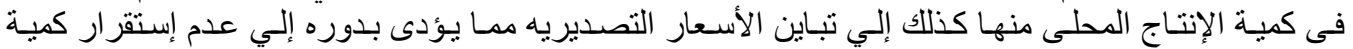

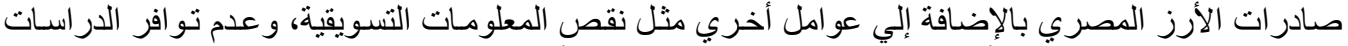

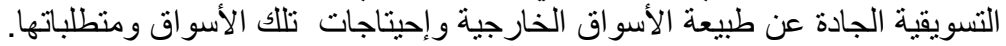

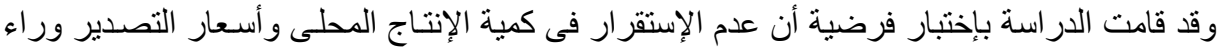

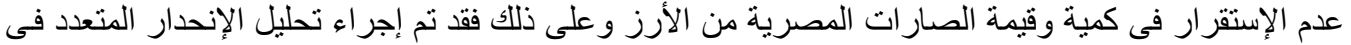

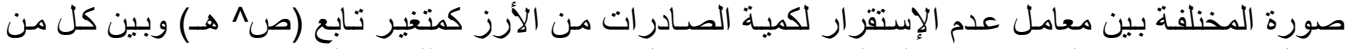

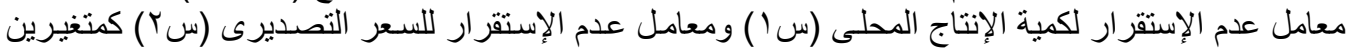

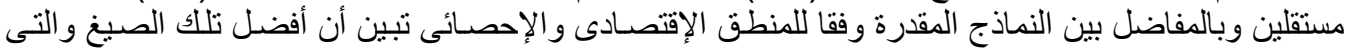

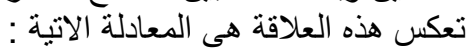

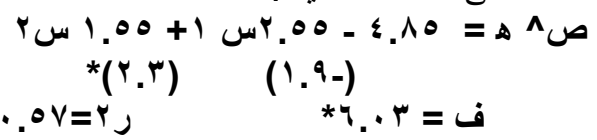

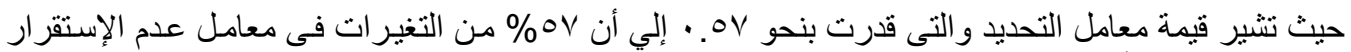

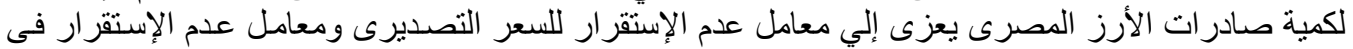

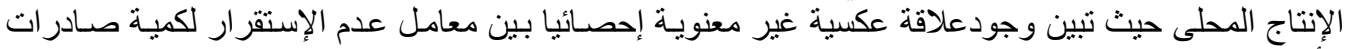

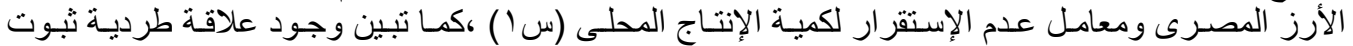

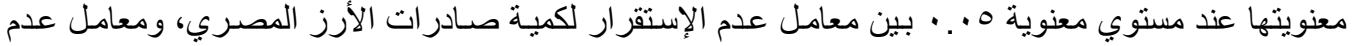

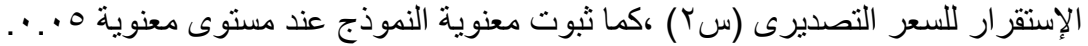

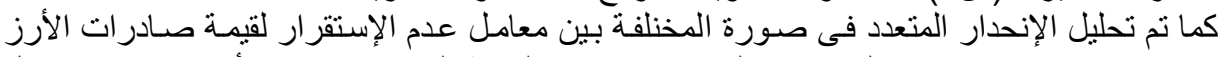

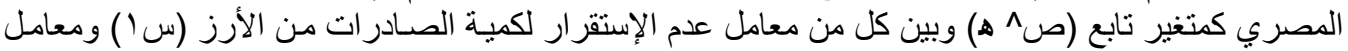

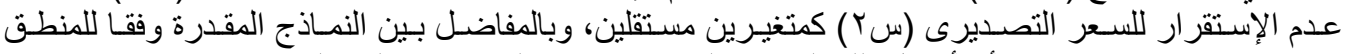

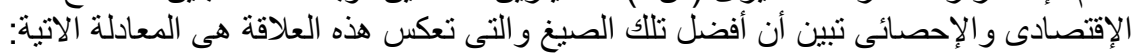

صـ A =

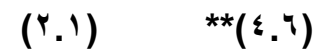

$$
\because A 0=r j \quad \text { ف }
$$

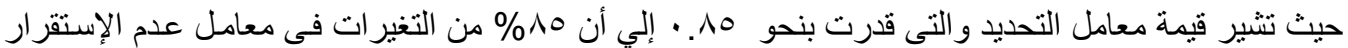

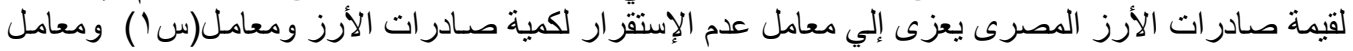

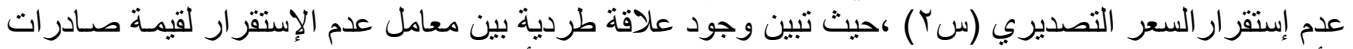

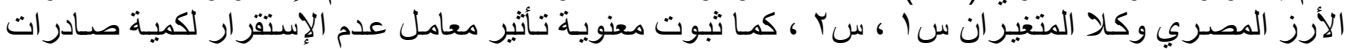

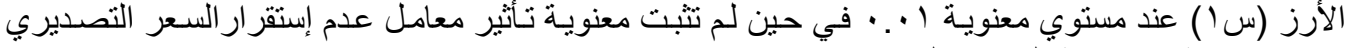

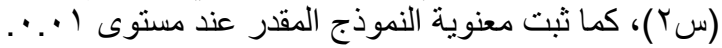

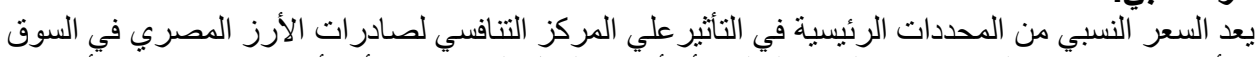

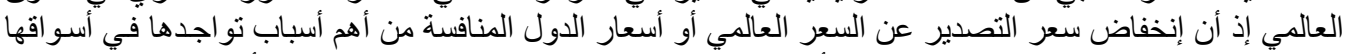

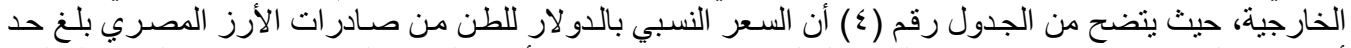

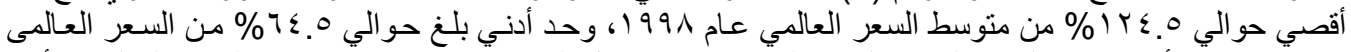

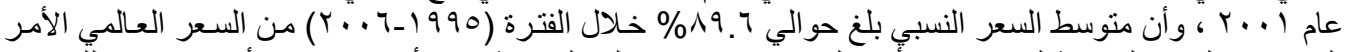

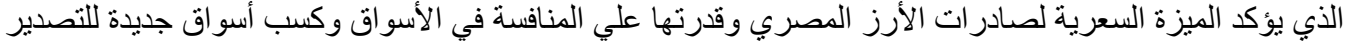

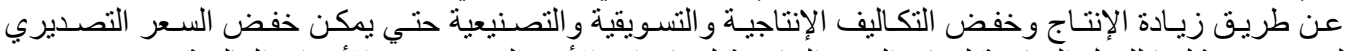

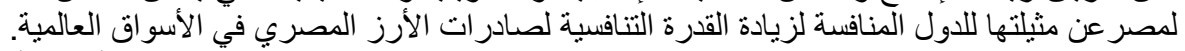

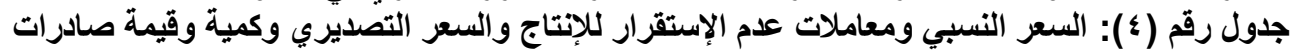

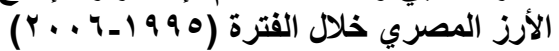

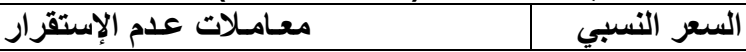
السنـة

Fayoum J. Agric. Res. \& Dev., Vol.23, No.1, January, 2009 
$\wedge$

\begin{tabular}{|c|c|c|c|c|c|}
\hline قيمة الصادرات & كمية الصادرات & سعر التصدير & الإنتاج المحلي & & \\
\hline$r \varepsilon . \varepsilon$ & $0 . . Y$ & $1 \leqslant . r$ & $\therefore \varepsilon$ & $9 Y .9$ & 1990 \\
\hline$\Lambda \cdot . \wedge$ & $\pi T^{2}$ & 10.4 & $\cdot . \wedge$ & NV.V & 1997 \\
\hline 14.0 & YV.O & $1 \leqslant .1$ & V.O & 94.1 & 1997 \\
\hline rT.r & $r \cdot . r$ & $\varepsilon .9$ & $1 . .0$ & $1 Y \leq .0$ & 1991 \\
\hline Y.. & rA. 9 & $\Gamma . \varepsilon$ & V.1 & 94. & 1999 \\
\hline $1 \leq .1$ & YY.T & $1 . r$ & V.Y & 1.9 .1 & r... \\
\hline $1 \Gamma . r$ & $r) . r$ & $1 . \varepsilon$ & 9.4 & $7 \leqslant .0$ & $r \ldots l$ \\
\hline$\varepsilon r_{.}$. & TY.乏 & 11.0 & r. & 9 qr. $^{2}$ & $r \ldots r$ \\
\hline ro. 9 & $Y . .0$ & $7 . Y$ & 1.5 & VI.1 & $r \ldots r$ \\
\hline \&.1 & IV.. & $r .9$ & 1.0 & 79.1 & $r \ldots \varepsilon$ \\
\hline 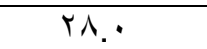 & YO.Y & $7 . \varepsilon$ & $\varepsilon . V$ & Nr.r & $r \ldots o$ \\
\hline $1 \leqslant 0$ & 1.1 & 19.4 & T.r & 9 9६. & $r \ldots r$ \\
\hline TV.乏 & rV.I & 9.1 & $\varepsilon .7$ & 19.07 & المتوسط \\
\hline
\end{tabular}

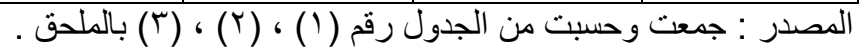

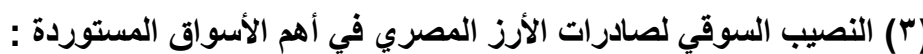

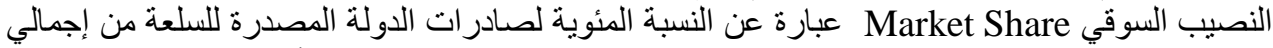

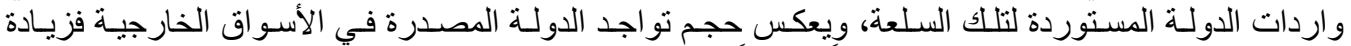

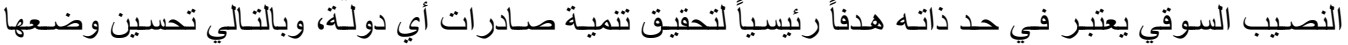

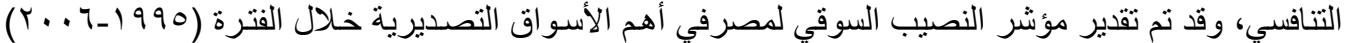

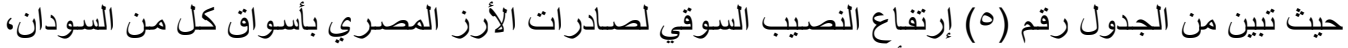

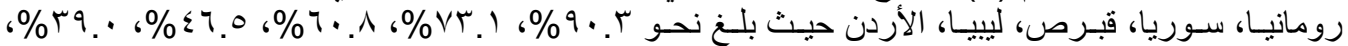

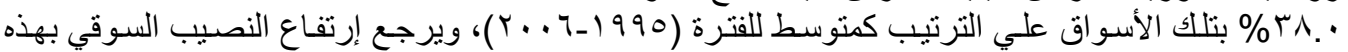

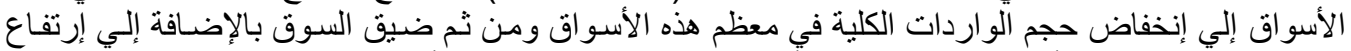

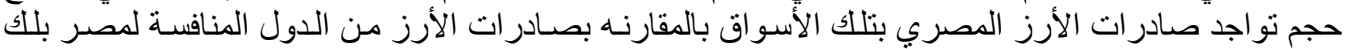

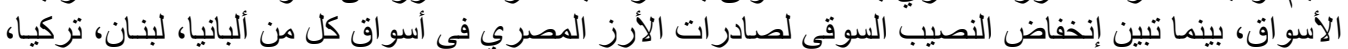

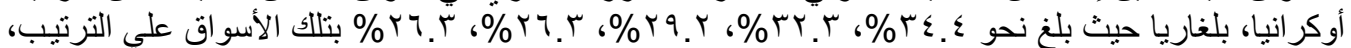

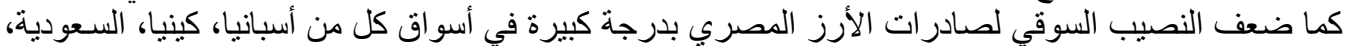

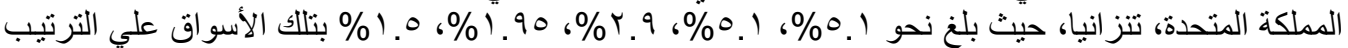
مما يعكس شدة المنافسة في تلك الأسو اق وضعف النصيب السوقي لصادرات أه الأرز المصري.

جدول رقم (•): مؤشرات النصيب السوقي ومعدل إختراق الأسواق في أهم الدول المستوردة للأرز المصري

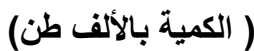

\begin{tabular}{|c|c|c|c|c|c|c|c|c|}
\hline تالدتيب & الختراق & تالدتيب & النوقي \% & صلادرات الدولة & اللاوليّي & الكلية للاودية & كمية واردات مصر من & الدول المستوردة وأهم \\
\hline \multicolumn{9}{|c|}{ (أ) أهم أسواق الدول العربية: } \\
\hline$r$ & 7.99 & $r$ & $7 . .1$ &..$\varepsilon$ &.$\cdot r$ & $I \vee \wedge . \varepsilon$ & $1 \cdot 1.0$ & 1- ا- سوريا \\
\hline 0 & rq.V & 7 & 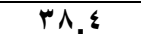 & T.7 & - & 111.2 & $\leqslant 5.9$ & با ب- الأردن \\
\hline$\Lambda$ & Tr.V & $\Lambda$ & rY.r & r.. & - & $\varepsilon \Lambda . r$ & 10.7 & 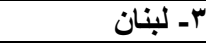 \\
\hline 1 & $V \leq . \varepsilon$ & 1 & $9 \cdot . r$ & - & V.Y & $r 4.9$ & $r \cdot .7$ & عـ ــ السودان \\
\hline 7 & $r 9.9$ & $0^{\circ}$ & $r 9$. &..$Y$ & - & 119.0 & $\$ 7.7$ & هـ ليبيا \\
\hline ir & Y.9 & $1 \varepsilon$ & 5.9 & 7.1 & - & VAT.r & YY.A & 1- السعودية \\
\hline (') & r... & (') & rI.V & $1 Y .1$ & V.ru & Irvo.1 & rVV.. & الجملة الجة \\
\hline
\end{tabular}

Fayoum J. Agric. Res. \& Dev., Vol.23, No.1, January, 2009 
$\wedge r$

\begin{tabular}{|c|c|c|c|c|c|c|c|c|}
\hline 1. & $1 \leqslant . \wedge$ & 1. & Y..ri & .9 & 00.9 & $v \cdot . v$ & 11.7 & 1- أوكرانيا \\
\hline 9 & IV.V & 11 & rצ.r. & - & $1 \leqslant .1$ & rq.r & $\mathrm{V} . \mathrm{V}$ & r - بلغاريا \\
\hline$r$ & 79.5 & $r$ & $V^{2} .1$ & $1 . \cdot$ & $\Lambda .7$ & $V 0 . Y$ & $00 .+$ & r- ب- رومانيا \\
\hline v & $r \varepsilon .0$ & v & $r \varepsilon . \varepsilon$ & $\because .0$ & - & $1 \wedge . r$ & $7 . r$ & ع ـ ألبانيا \\
\hline 11 & $1 Y .7$ & 9 & rq. & 1.9 & $r 9 . r$ & $r V \cdot . \Lambda$ & Vq. & 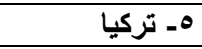 \\
\hline$(Y)$ & $1 \cdot .1$ & $(Y)$ & $10 . r$ & r.q & $\leqslant \mu q . \wedge$ & $11 \ldots .7$ & 177.1 & الجملة \\
\hline \multicolumn{9}{|c|}{ (ج) أهم أسواق دول أورويا الغربية : } \\
\hline 10 & .9 & Ir & 0.11 & $r \wedge 1 . V$ & $V \leq V . V$ & $1 \cdot V . Y$ & 0.0 & 1- أسباتيا \\
\hline $1 \varepsilon$ & Y. & 10 & 1.9 & $r \wedge . \wedge$ & - & $\varepsilon r 7.9$ & $\Lambda . \varepsilon$ & بـ المملكة المتحدة \\
\hline$\varepsilon$ & ะ४.V & $\varepsilon$ & $\leqslant 7.0$ & $\cdot . r$ & - & $\varepsilon . \mu$ & Y.P & " بـ قبرص \\
\hline$(r)$ & 1.7 & $(\xi)$ & Y. 9 & $r Y \cdot .0$ & $V \leq V . V$ & $0 \leqslant \Lambda . \leqslant$ & 10.9 & الجملة \\
\hline \multicolumn{9}{|c|}{ (د) أهم أسواق دول أفريقيا : } \\
\hline 17 &..$\varepsilon$ & 17 & 1.0 & 7.1 & OYV.r & IVะ.. & r. & 1ـ تتزانيا \\
\hline Ir & $\varepsilon . \cdot$ & 14 & 0.1 & $r . r$ & $\varepsilon \varepsilon .7$ & $1 \leqslant V .9$ & $\mathrm{~V} .0$ & r- كينيا \\
\hline$(\xi)$ & 1.1 & $(r)$ & $r .1$ & 9.8 & $0 \vee 1 . \Lambda$ & rYI.9 & $1 \cdot .1$ & الجملة \\
\hline- & - & - & - & - & - & - & $0 \leq 7 . r$ & إلجمالي صصادرات المصري \\
\hline
\end{tabular}

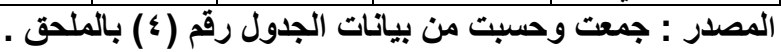

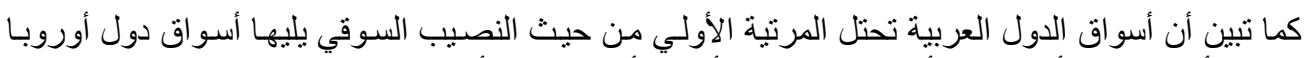

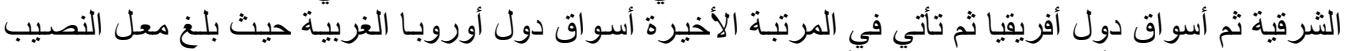

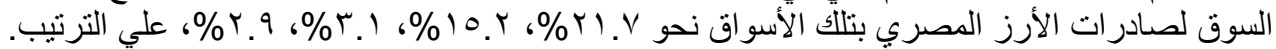

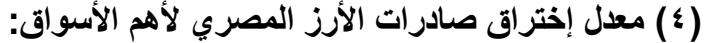

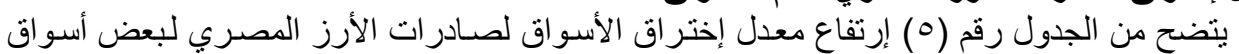

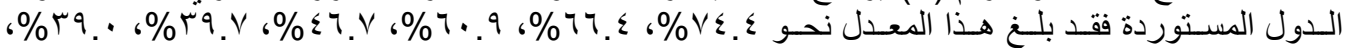

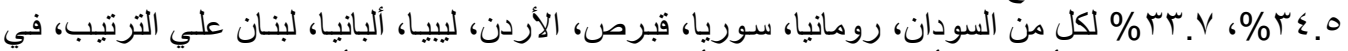

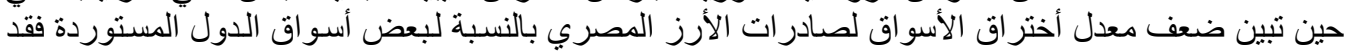

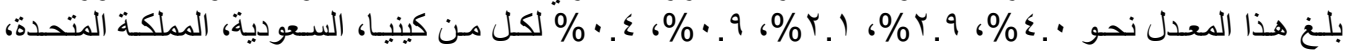

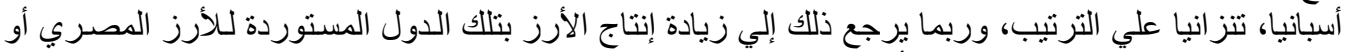

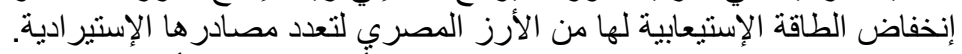

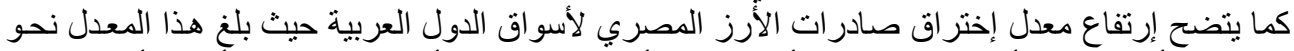

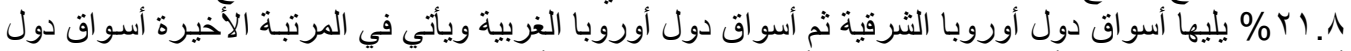

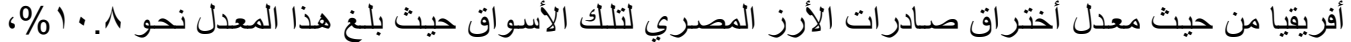

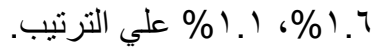
إلملخص والتوصيات:

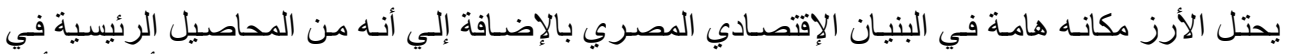

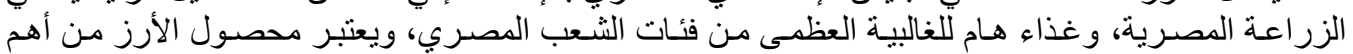

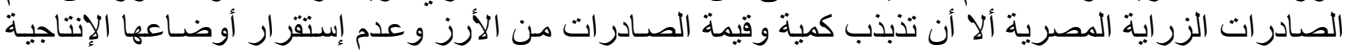

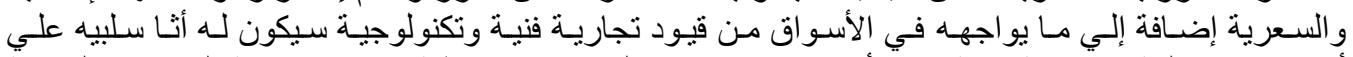

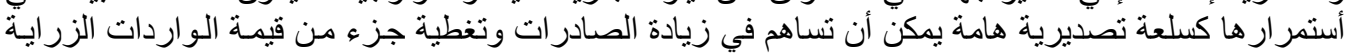

المتتامية.

ويهدف البحث بصفه أساسية إلي إلقاء الضـوء علي صـادر ات الأرز المصري من كن حيث در اسـة الأهميـة

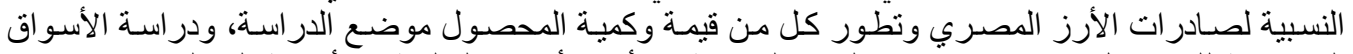

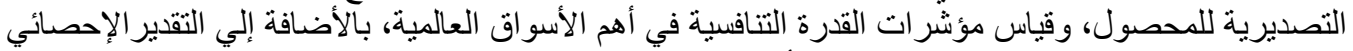

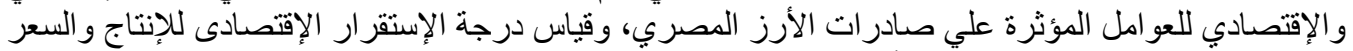

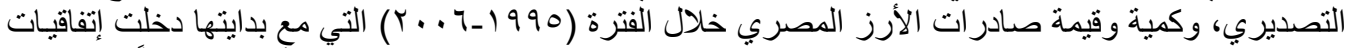

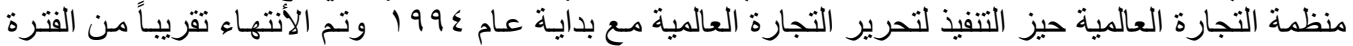

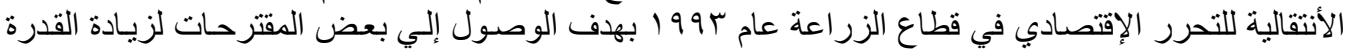

التنافية في الأسواق التصدية الأصديرية

Fayoum J. Agric. Res. \& Dev., Vol.23, No.1, January, 2009 
$\Lambda \boldsymbol{H}$

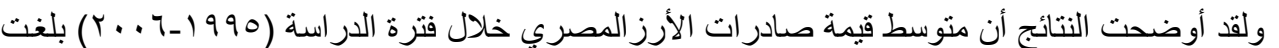

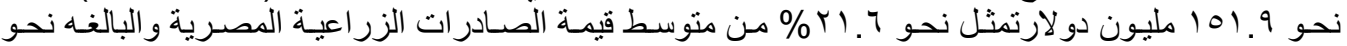

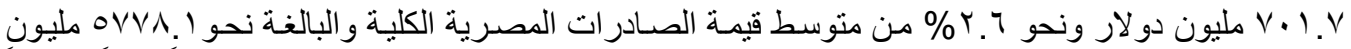

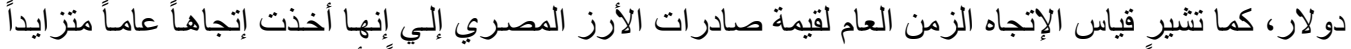

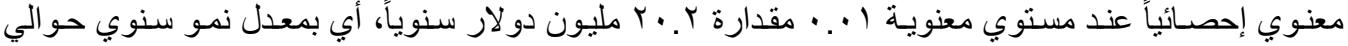

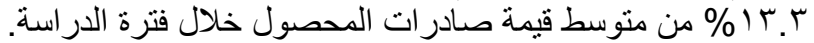

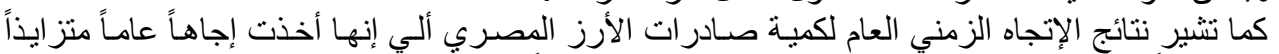

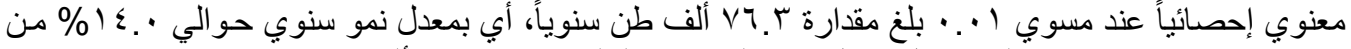

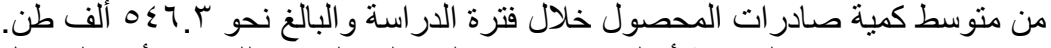

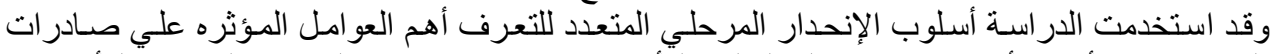

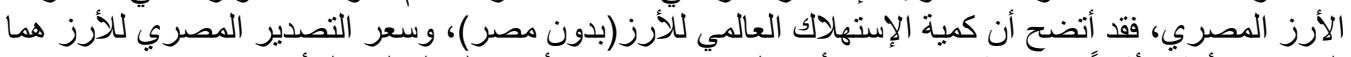

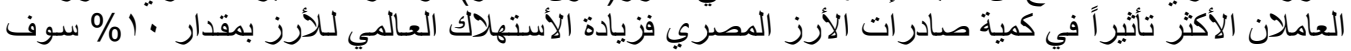

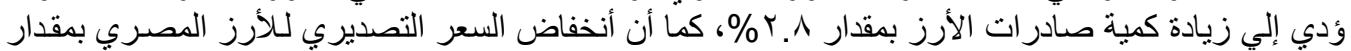

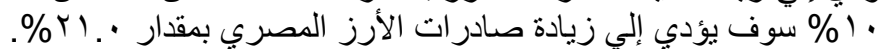

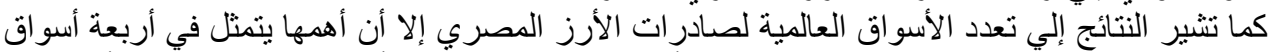

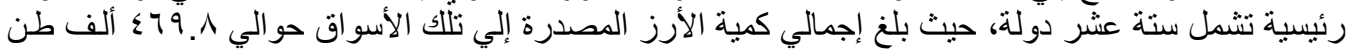

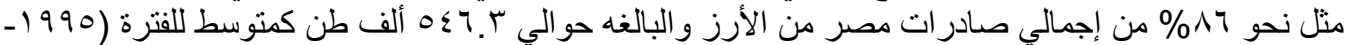

. ( Y.. T

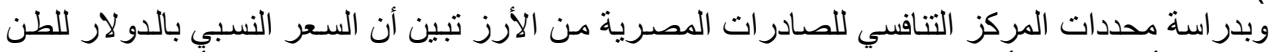

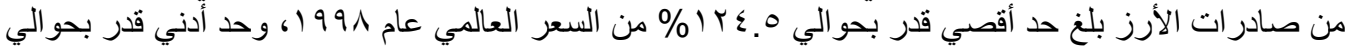

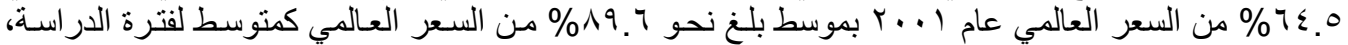

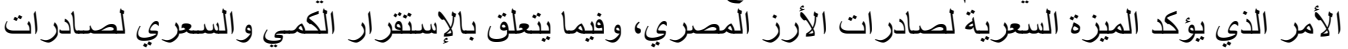

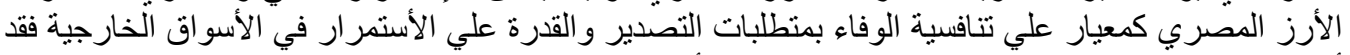

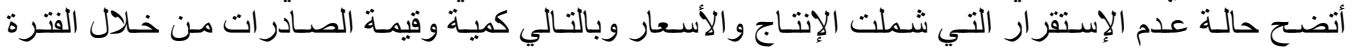

(Y... T-1990)

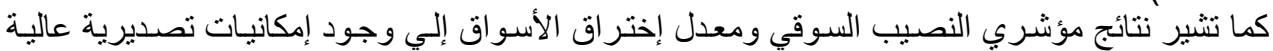

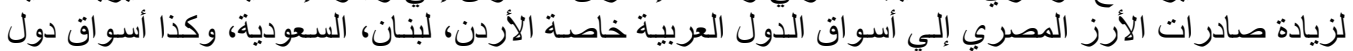

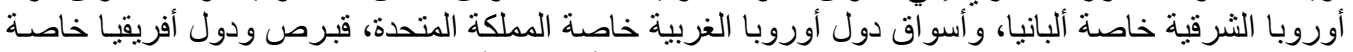

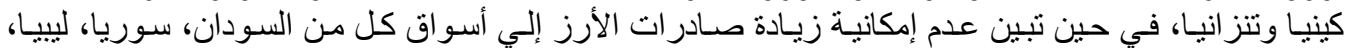
أوكر انيا، بلغاريا، تركيا، وأسبانيا.

\section{وعليه فإن الاراسة توصي بالأتي :}

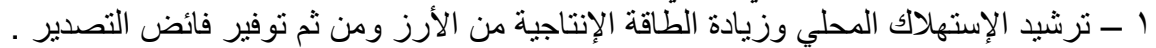

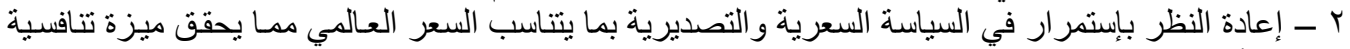

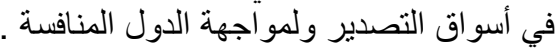

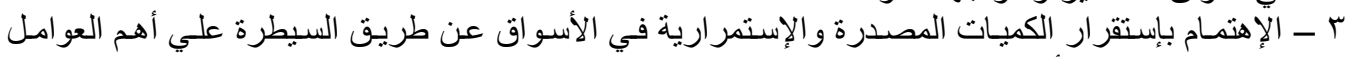

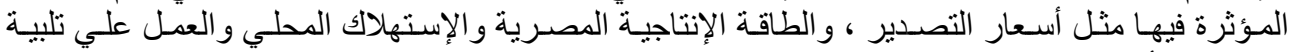

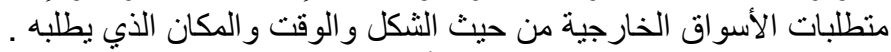

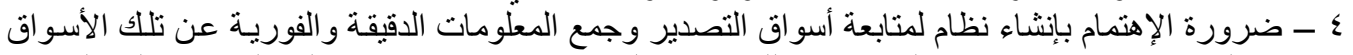

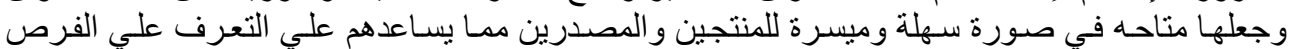

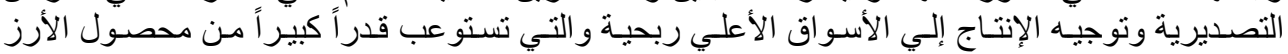
المصري.

ه ـ نظر اً لأهمية الأسواق العربية وأسواق تركيا ودول شرق أوروبا يجب العمل علي الإستفادة من الإتفاقيات

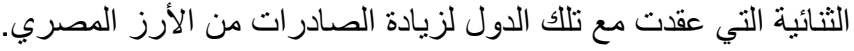

المراجـع: (الم:ع

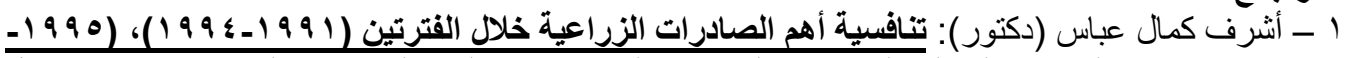

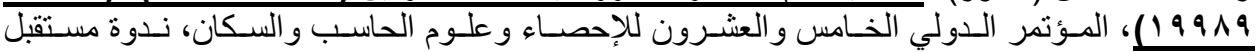

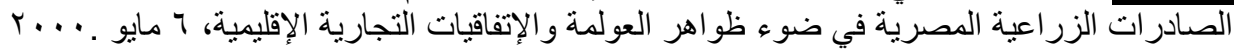

Fayoum J. Agric. Res. \& Dev., Vol.23, No.1, January, 2009 
r - الجهاز المركزي للتعبئة العامة والإحصاء: النشرة السنوية للتجارة الخارجية، أعداد متفرقة.

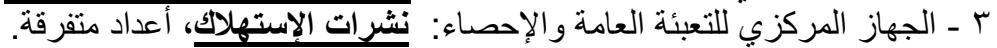

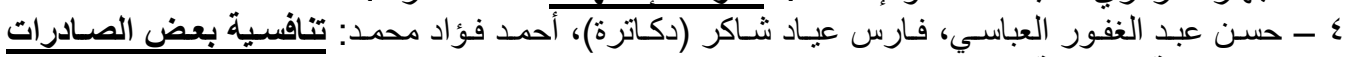

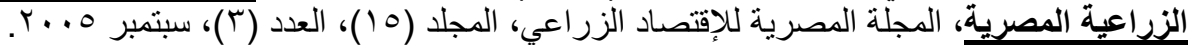

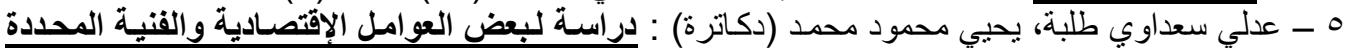

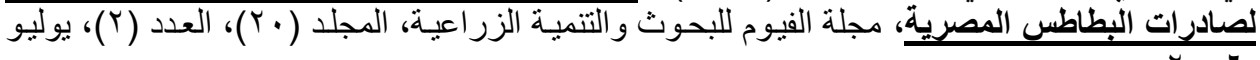

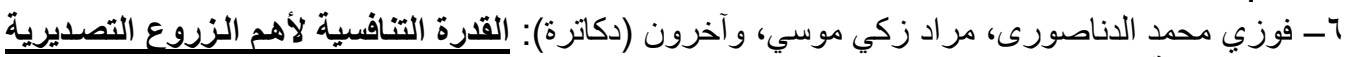

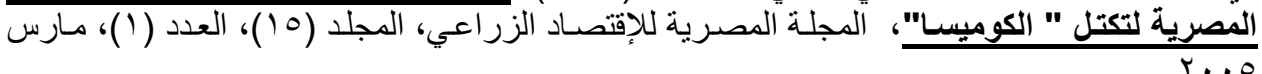

V - مصطفي عبد ربه القبلاوي: القدرة التنافسية لصادرات الأرز المصري في أهـ الهم الأسواق العالمية، المجلة

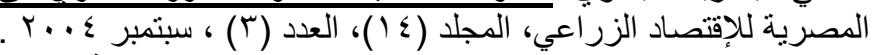

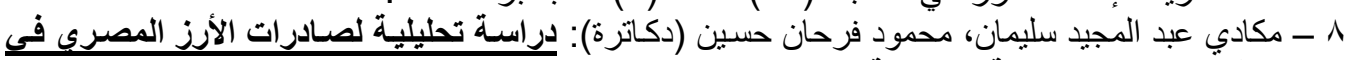

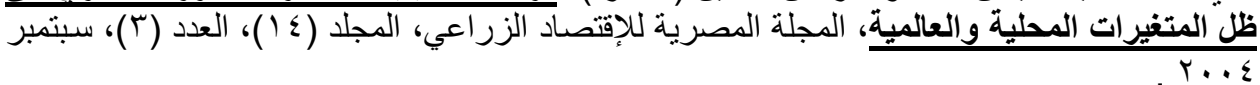

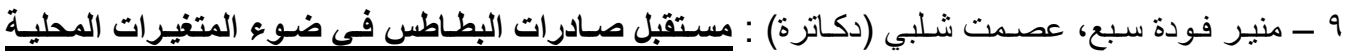

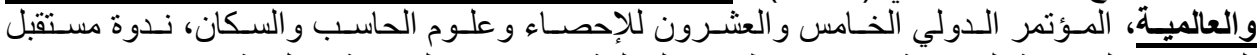

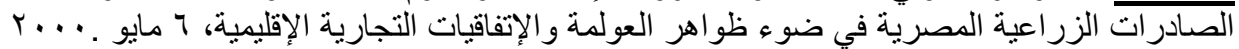

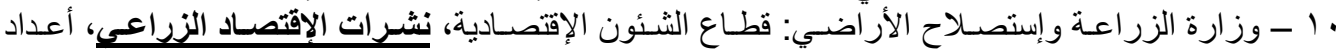

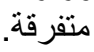

1) - موقع منظمة الأغذية و الزر اعة علي الثبكة الدولية للمعلومات: Www.fao.org

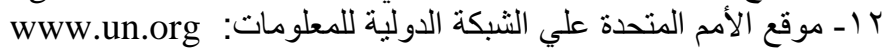

13-Foreign Agricultural service /USDA, office of fice of Global analysis, April, 2007.

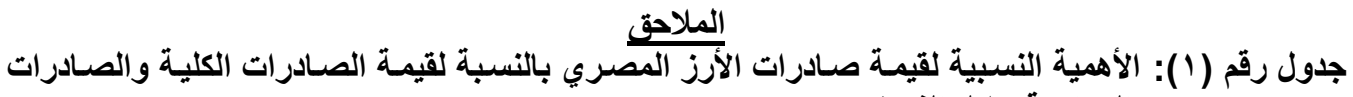

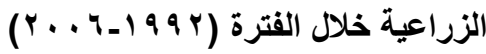

القيمة بالمليون دولار

\begin{tabular}{|c|c|c|c|c|c|c|}
\hline \multicolumn{3}{|c|}{ صادرات الأرز } & \multicolumn{2}{|c|}{ الصادرات الزراعية } & \multirow{2}{*}{ قيمة الصلية } & \multirow[b]{2}{*}{ 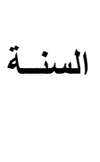 } \\
\hline الزادرات & الصادرات الكلية & قيمة صادرات & الصادرات الكلية & قيمة الصادراتية & & \\
\hline $1 \leqslant . r$ & Y.. & 00. & 17.9 & $\leqslant \wedge 1$. & $r \leqslant 0 .$. & 1990 \\
\hline YT.V & $r . r$ & $11 . .0$ & $1 \varepsilon .1$ & $\varepsilon 9 \Lambda_{.}$. & rorq. & 1999 \\
\hline$Y \Lambda .1$ & r.r & $v 1.1$ & 11.5 & $\varepsilon r q_{.}$. & rqץl.• & $199 \mathrm{~V}$ \\
\hline Y५.V & \&.r & Tro.r & 17.5 & $0 . v_{.}$. & r/r... & 1991 \\
\hline 17.7 & Y.O & 19.9 & $1 \leqslant . \wedge$ & OrV. & rooq.. & 1999 \\
\hline$Y \cdot .0$ & Y.Y & 141.9 & $1 \cdot . \Lambda$ & 0.1. & 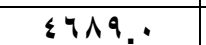 & $r \ldots$ \\
\hline Y 9.4 & T.0 & $1 \varepsilon \cdot . r$ & $11 . \wedge$ & $\leqslant 90$. & $\leqslant I \vee V_{.}$ & $r \ldots l$ \\
\hline $1 \leqslant .0$ & T.Y & 1.0 .7 & 17.1 & VOr. & $\varepsilon \neg \vee \Lambda_{.}$. & $r \ldots r$ \\
\hline 19.0 & Y. & $10 .$. & $1 \varepsilon . V$ & 9.9 .9 & 7171. & $r \ldots r$ \\
\hline 11.1 & r.. & rTI.V & 19. & TYYY. & จฯง . & $r \ldots \varepsilon$ \\
\hline$Y \Lambda_{.} \Lambda$ & Y.q & $M 1 . .0$ & $1 \cdot .1$ & 1.89. & $1 . Y \leqslant Y$. & $r \ldots o$ \\
\hline
\end{tabular}

Fayoum J. Agric. Res. \& Dev., Vol.23, No.1, January, 2009 


\begin{tabular}{|c|c|c|c|c|c|c|}
\hline$r \cdot . r$ & $Y . Y$ & r...q & $V . r$ & 990.0 & I rvo... & $r \ldots r$ \\
\hline Y1. & $Y .7$ & 101.9 & $1 Y .1$ & $v .1 . V$ & 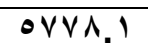 & المتوسط \\
\hline
\end{tabular}

المصدر: جمعت وحسبت من الجهاز المركزي للتعبئة العامة والإحصاء: النشرة السنوية للتجارة الخارجية ، أعداد متفرقة.

جدول رقم (r): تطور الإتتاج العـالمي والمصري لمحصول الأرز والأهمية النسبية لصسادرات الأرز المصري بالإلنسية الكمية بالألف طن بري

\begin{tabular}{|c|c|c|c|c|c|c|c|}
\hline الإلتاج من & صادر من الأرز & صادرات & صادرات الأرز & الإنتاج من & اللألصرى الأتتاج & العالمي للأرز & السنة \\
\hline \&. &.$\wedge$ & $19 \cdot . r$ & rrol... & $\because .9$ & $\varepsilon \vee \wedge \wedge$. & $0 \leqslant V \leqslant Y Y_{.} \cdot$ & 1990 \\
\hline $7 . V$ & $1 . V$ & 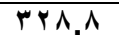 & $19 V 4 \cdot$. & $\cdot .9$ & $\varepsilon \wedge 90^{\circ}$ & $0 ૫ \wedge 9.0 . \wedge$ & 1997 \\
\hline 7.8 & $1 . V$ & $Y \cdot Y . Y$ & Y.9人7. & $\cdot .9$ & $0 \leqslant \wedge .$. & 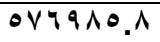 & 1998 \\
\hline 9.7 & 1.0 & $\varepsilon Y q . Y$ & $r \wedge \wedge \varepsilon r$. & $\cdot . \wedge$ & $\leqslant \leqslant 0 .$. & 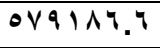 & 1991 \\
\hline $0 . r$ & $1 . r$ & $r \cdot v_{.} \cdot$ & $r \otimes r \leqslant 9 . *$ & $\cdot .9$ & $0 \wedge 17$. & $71.9 \wedge \wedge .9$ & 1999 \\
\hline 7.1 & 1.0 & Mчr.A & rrooq. & $1 .$. & $7 \ldots$. & 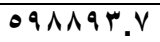 & $r \ldots$ \\
\hline $1 \% .0$ & Y. & $\vee \cdot \Lambda . \vee$ & $r \checkmark \wedge r V$. & $\because .9$ & OYYV.. & $\Delta ৭ \wedge . r \Delta .0$ & 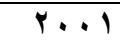 \\
\hline 8.9 & $1 . v$ & $\varepsilon \Lambda \cdot . r$ & rVRVI.. & 1.1 & 71.0. & O१৭YYA.• & $r \ldots r$ \\
\hline 9.0 & Y. & $\bullet \wedge \bullet . \wedge$ & 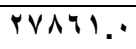 & 1.1 & $71 \vee \leqslant$. & $\Delta \wedge \leqslant \Lambda Y Y . \theta$ & $r \ldots r$ \\
\hline 14.1 & Y. 1 & ATr.\& & 19.97. & 1.9 & 7401. & $7 . v \vee \leq 1 . \varepsilon$ & $r \ldots \varepsilon$ \\
\hline $11 . r$ & r.A & $111 \% .8$ & rqrrr.. & $1 . \cdot$ & $71 Y \leq$. & 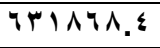 & $r . .0$ \\
\hline $1 \varepsilon .7$ & $r . r$ & q^r.ะ & $r \cdot r \cdot q . \cdot$ & $1 .$. & $7 V \leq \varepsilon$. & $7 \leq \leqslant 110$. & $r . .7$ \\
\hline 9.7 & Y.. & $0 \leq 7 . r$ & rqVav.^ & .99. & $07 \vee 9.0$ & OqMIAr.q & المتوسط \\
\hline
\end{tabular}

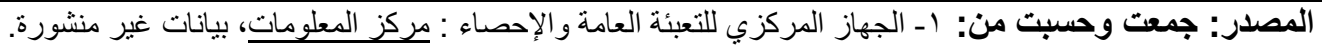

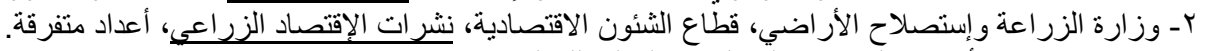

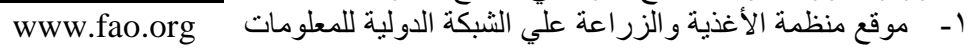

جدول رقم (ب): تطور كميـة الإسـتهلاك والمخزون والسـعر التصـيري والمسـاحة المنزرعـة بـالأرز علـي

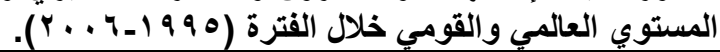

\begin{tabular}{|c|c|c|c|c|c|c|c|}
\hline \multicolumn{2}{|c|}{ التصدير(دولار/طن) } & \multicolumn{2}{|c|}{ المساحـة المنزرعة } & \multirow{2}{*}{ 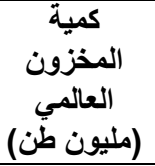 } & \multicolumn{2}{|c|}{ كميـة الاستهلاك } & \multirow[t]{2}{*}{ السنة } \\
\hline المصري & العالمي & (ألف فصران) & (مليون هكتار) & & القومي (ألف & (مليون طن) & \\
\hline$r \wedge 9 . \cdot$ & M1.. & $1 \varepsilon \cdots$ & $1 \leqslant \Lambda_{.} \cdot$ & 111.0 & $r 017 . r$ & ryะ.7 & 1990 \\
\hline rrq. & r^r.. & $1 \leqslant .0 .0$ & $10 . .0$ & $111 .+$ & YOTY.E & rqv. & 1997 \\
\hline rol." & $r v v^{\prime} \cdot$ & $10 \leqslant 9.0$ & 101.0 & $1 Y 1.0$ & rOYY. & $r \vee \wedge .7$ & $199 V$ \\
\hline Mlo.. & ror.. & Irro.. & 10\%.. & IY & YYTะ.7 & rVq.0 & 1991 \\
\hline rqr.. & M10. & 1009. & 100.0 & $1 T \varepsilon$. & Y & $r \wedge V_{.0}$ & 1999 \\
\hline$r 1 .$. & $Y \wedge \leq$. & 1079.0 & $10 Y . \cdot$ & $1 \leq 0.0$ & $\mu .1 \Lambda .0$ & rqv.s & $r \ldots$ \\
\hline 191.0 & $r . v . \cdot$ & $1 \Gamma \leqslant \cdot$. & 101.0 & $1 \leqslant 9$. & YIIr. & rqs. & $r \ldots 1$ \\
\hline$r r \cdot . \cdot$ & rrq." & $10 \leqslant V_{.0}$ & $1 \leq 7$. & $1 \% 7$. & $r .11 .0$ & $\{11.1$ & $r \ldots r$ \\
\hline Y04." & $r 4 .$. & 10.1 .0 & $1 \leqslant \Lambda_{.} \cdot$ & $1 . v . \cdot$ & 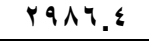 & $\varepsilon \cdot 7 . r$ & $r \ldots r$ \\
\hline rVA. & $\varepsilon \cdot r_{.} \cdot$ & $10 r V . \cdot$ & $10 \cdot$. & ^०.. & \&Y79. & $\varepsilon 1 \%$. & $Y \cdots \varepsilon$ \\
\hline rvq.. & rro.s & $1 \leqslant 09.0$ & $10 \%$. & $\vee \wedge_{.} \cdot$ & $\varepsilon \wedge \neg \wedge$. & $\varepsilon \cdot \Lambda_{.} \cdot$ & $r \ldots o$ \\
\hline$r . q^{*}$ & rro.. & 1094. & $10 \%$. & $\Lambda r_{.} \cdot$ & 0.97. & $\leqslant 10$. & $r \ldots q$ \\
\hline$r \wedge 0.9$ & $r r \leq . r$ & $1 \leqslant V \leqslant . r$ & $10 \cdot .1$ & 117.8 & 191.1 & rq4.7 & المتوسط \\
\hline
\end{tabular}

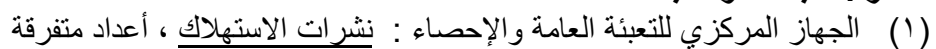

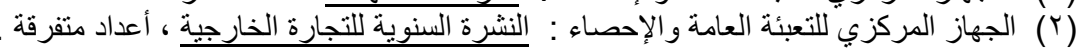

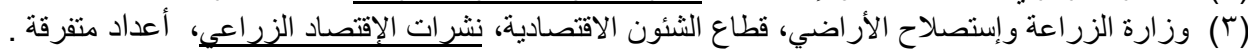

Fayoum J. Agric. Res. \& Dev., Vol.23, No.1, January, 2009 
ᄉ

(4) Foreign Agricultural Service / USDA , Office of Global analysis , April , 2007.

جدول رقم (؛ ) تطور واردات وإنتاج وصادرات الأرز بالألف طن في أهم الدول المستوردة للأرز المصري الكمية بالألف طن

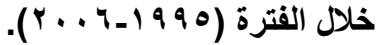

\begin{tabular}{|c|c|c|c|c|c|c|c|c|c|c|c|c|}
\hline \multicolumn{3}{|c|}{ 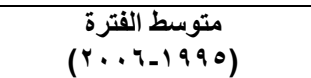 } & \multicolumn{3}{|c|}{ 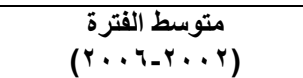 } & \multicolumn{3}{|c|}{ 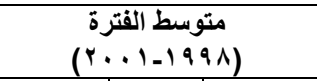 } & \multicolumn{3}{|c|}{ 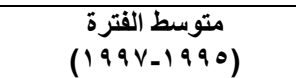 } & \multirow[t]{2}{*}{ الدولة الدي } \\
\hline صادرات & إنتاج & واردات & | & إنتاج & واردات & صادرات & إنتاج & واردات & | صادرات & إنتاج & واردات & \\
\hline \multicolumn{13}{|c|}{ أ - الدول العربية: } \\
\hline$\cdot . \varepsilon$ &..${ }^{\top}$ & IV^. $\varepsilon$ & .0 & - & Y.7.9 & $\cdot . \mathrm{V}$ & - & 170.1 & $\because \cdot r$ & $\because . \wedge$ & 174.0 & سوريا \\
\hline$r .7$ & - & 111.8 & 1.7 & - & $1 Y \leqslant . V$ & V.V & - & $1 \cdot r .1$ & 1.0 & - & $1 \cdot 8.7$ & الأردن \\
\hline Y." & - & $\varepsilon \wedge . r$ & $r .7$ & - & Or. & 1.9 & - & $\{7.1$ & $\cdot . \varepsilon$ & - & $\$ 7 . \wedge$ & لبنان \\
\hline - & V.r & rr.q & - & IT. & $\varepsilon \Lambda_{.}$. & - & A.. & 51.9 & - & $1 . v$ & $1 \Lambda . V$ & السودان \\
\hline$\because \cdot Y$ & - & 119.0 & 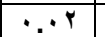 & - & $1 r 0.9$ & $\because . r$ & - & $1 \times 9.7$ & - & - & 1.4 .9 & لييا \\
\hline 7.1 & - & V^r.r & 7.8 & - & $\Lambda ฯ \Lambda .9$ & $r .7$ & - & AMI.r & $\Lambda . r$ & - & $7 \leq 9.1$ & السعودية \\
\hline \multicolumn{13}{|c|}{ ب - دول شرق أوروبا: } \\
\hline$\cdot .9$ & 00.9 & $V \cdot . v$ & $\cdot . r$ & 19.9 & 99.5 &.$r$ & $V 1 . \varepsilon$ & 79. & Y.r & $V 7.0$ & $\leqslant 7.9$ & أوكرانيا \\
\hline- & $1 \leq .1$ & YQ.r & - & rY.. & $r \cdot .9$ & - & IT.Y & YO.\& & - & V.1 & $\$ 1.0$ & بلغاريا \\
\hline $1 . \cdot$ & $\Lambda .7$ & VO.Y & $\because . r$ & \&. & $9 \cdot . r$ & 1.2 & $r .0$ & $\Lambda 1.1$ & $1 . r$ & $1 \Lambda . r$ & $0 \leqslant . Y$ & رومانيا \\
\hline$\because .0$ & - & $1 \Lambda . r$ & $\because \cdot Y$ & - & rY.. & $\because \cdot r$ & - & $11 . r$ & $\because 1$ & - & $1 \leq . V$ & ألبانيا \\
\hline 1.9 & $r 9 . r$ & $r v \cdot . \Lambda$ & Y.P & 0.4 .7 & r^r.^ & r.. & $r \leqslant 1 . r$ & YVY.Z & $\cdot . \wedge$ & rrA.V & rOV.r & تركيا \\
\hline \multicolumn{13}{|c|}{ ج - دول غرب أوروبا: } \\
\hline rAI.V & $V \leqslant V . V$ & $1 . V . r$ & $r \leqslant r . r$ & $\Lambda \leq 0 . r$ & $91 . r$ & pqY.q & Ar५. & A.. & 4.9 .1 & 701.1 & $1 \leq \varepsilon$. & أسباتيا \\
\hline$r \wedge . \wedge$ & - & $\varepsilon r 4.9$ & \&.. & - & 0.7.1 & $\$ 1.9$ & - & $\varepsilon Y 0 . \varepsilon$ & $r \wedge .7$ & - & $r \leqslant q . r$ & |المملكة المتحدة \\
\hline$\because \cdot Y$ & - & $\varepsilon . r$ & $\because \cdot 1$ & - & $0 . r$ & $\because \cdot Y$ & - & $\varepsilon . Y$ & $\because \cdot \varepsilon$ & - & r.o & قبرص \\
\hline \multicolumn{13}{|c|}{ د - دول أفريقيا } \\
\hline 7.1 & OYV.Y & IVะ.. & A. $\cdot$ & 1.19 .8 & $r .1 .1$ & $1 \cdot . \cdot$ & 119.0 & $1 \leq 9.8$ & $\cdot . r$ & 709.8 & $v 1.7$ & تنزانيا \\
\hline$r . r$ & $\leqslant \leqslant .7$ & $1 \leq V .9$ & $\because 0$ & 0 O.० & $r 1 \leq . V$ & $\cdot r$ & $r 0.0$ & A9.V & 9.1 & $\leqslant 0 . V$ & $r 9.8$ & كينيا \\
\hline
\end{tabular}

Fayoum J. Agric. Res. \& Dev., Vol.23, No.1, January, 2009 


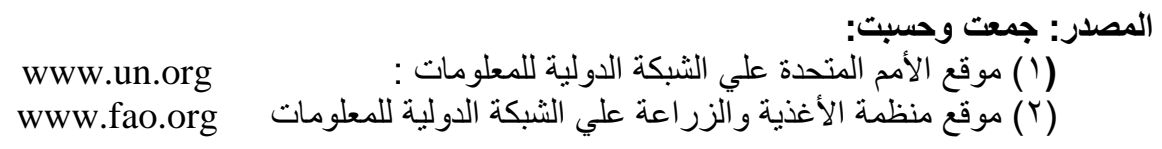

\title{
COMPETITIVE CAPACITY OF EGYPTIAN RICE EXPORTS UNDER THE ECONOMIC REFORM POLICY
}

\author{
Alaa Ahmed Kotb and Gamal Alsayed Mohamed \\ Agricultural Economics Research Institute
}

\section{Summery}

The study aims at highlighting the Egyptian rice exports. It highlights the relative importance of the Egyptian rice exports, the development of the quantity and value of rice exports, and investigating the competitive capacity within the international markets. It also investigates the statistical and economic estimations of the factors affecting the Egyptian rice exports and measures the economic stability of production, prices, and quantity and value of the exports during the period 1995-2006. The previously mentioned objectives will lead to recommendation for improving the competitive capacity in the international markets.

The study has shown the following results:

1- The trends of the quantity and value of the Egyptian rice exports are increasing by 76300 tons and $\$ 20.2$ millions with and annual growth of $14 \%$ and $13.3 \%$ respectively.

2- Egypt has a price competitive advantage where the average of relative price for ton in US dollars comparing to the world price, as an average of the study period, was $89.6 \%$.

3- There is a high fluctuation and instability of the quantity and quality of the rice exports where the instability ratios for both indicators were estimated as $27.1 \%$ and $27.4 \%$ respectively. The average of instability ratio of local production and

Fayoum J. Agric. Res. \& Dev., Vol.23, No.1, January, 2009 
export price of rice were $4.6 \%$ and $9.1 \%$ respectively during the period 1995 2006.

4- The results refer to the variation of Egyptian rice exporters' markets, in particular 16 markets, the total quantity exported to these markets were about 496800 tons represents about $86 \%$ of the total exports of rice which is about 546300 tons as an average of the period 1995-2006. By estimating the Gini Hirchman ratio of geographical concentration of the Egyptian rice exports, it's shown that he ratio is about $57.2 \%$ as an average of the period 1995-2006 which means that the rice exports is concentrated within certain markets since the ratio is more than $40 \%$ according to Hirchman.

5- The market share and market penetration ratios were estimated where the Sudanese market comes in the first rank followed by the Romanian, Syrian, and Cyprus Markets respectively. 